\title{
Identification of a motif that mediates polypyrimidine tract-binding protein-dependent internal ribosome entry
}

\author{
Sally A. Mitchell, ${ }^{1,3}$ Keith A. Spriggs, ${ }^{1,3}$ Martin Bushell, ${ }^{1}$ Joanne R. Evans, ${ }^{1}$ Mark Stoneley, ${ }^{1}$ \\ John P.C. Le Quesne, ${ }^{1}$ Ruth V. Spriggs, ${ }^{2}$ and Anne E. Willis ${ }^{1,4}$ \\ ${ }^{1}$ School of Pharmacy, University of Nottingham, University Park, Nottingham, NG7 2RD, UK; ${ }^{2}$ European Bioinformatics \\ Institute, Wellcome Trust Genome Campus, Hinxton, Cambridge, CB10 1SD, UK
}

\begin{abstract}
We have identified a novel motif which consists of the sequence $(\mathrm{CCU})_{\mathbf{n}}$ as part of a polypyrimidine-rich tract and permits internal ribosome entry. A number of constructs containing variations of this motif were generated and these were found to function as artificial internal ribosome entry segments (AIRESs) in vivo and in vitro in the presence of polypyrimidine tract-binding protein (PTB). The data show that for these sequences to function as IRESs the RNA must be present as a double-stranded stem and, in agreement with this, rather surprisingly, we show that PTB binds strongly to double-stranded RNA. All the cellular $5^{\prime}$ untranslated regions (UTRs) tested that harbor this sequence were shown to contain internal ribosome entry segments that are dependent upon PTB for function in vivo and in vitro. This therefore raises the possibility that PTB or its interacting protein partners could provide a bridge between the IRES-RNA and the ribosome. Given the number of putative cellular IRESs that could be dependent on PTB for function, these data strongly suggest that PTB-1 is a universal IRES-trans-acting factor.
\end{abstract}

[Keywords: Polypyrimidine tract-binding protein; IRES; internal ribosome entry; translation; artificial IRES]

Supplemental material is available at http://www.genesdev.org.

Received February 1, 2005; revised version accepted May 19, 2005.

Initiation of protein synthesis can occur by two distinct mechanisms: cap-dependent scanning and internal ribosome entry. The former mechanism, which is used by the majority of mRNAs, requires the binding of the eukaryotic initiation factor complex $4 \mathrm{~F}$ (eIF4F) to the 7-methyl-G cap structure at the $5^{\prime}$ end of an mRNA, recruitment of the $40 \mathrm{~S}$ ribosomal subunit, and scanning to the first AUG codon that is in the correct context (Pain 1996). The latter mechanism requires a structural element to be formed in the $5^{\prime}$ untranslated region (UTR) of the message, termed an internal ribosome entry segment (IRES), which, in the presence of IRES trans-acting factors (ITAFs), allows direct recruitment of the ribosome (Hellen and Sarnow 2001). This mechanism was first described for the picornavirus family, where it allows continued synthesis of viral proteins when cap-dependent scanning is reduced by viral modification of eIFs (Belsham and Jackson 2000). However, it has been shown

\footnotetext{
${ }^{3}$ These authors contributed equally to this work.

${ }^{4}$ Corresponding author.

E-MAIL anne.willis@nottingham.ac.uk; FAX 0115-951535.

Article and publication are at http://www.genesdev.org/cgi/doi/10.1101/ gad.339105.
}

that some cellular mRNAs can also initiate translation by internal ribosome entry. The majority of mRNAs that contain IRESs encode proteins that are involved in the regulation of cell growth, cell death, or cell stress, and internal ribosome entry provides a mechanism for the synthesis of these proteins under these conditions (Hellen and Sarnow 2001). In agreement with this it has been shown that internal ribosome entry is used during pathophysiological conditions where cap-dependent scanning is reduced, including following viral infection (Johannes et al. 1999), during apoptosis (Stoneley et al. 2000a), heat shock (Coldwell et al. 2001), hypoxia (Huez et al. 1998; Stein et al. 1998), genotoxic stress (Subkhankulova et al. 2001), and mitosis (Qin and Sarnow 2004). Possibly as many as $10 \%$ of cellular mRNAs contain IRESs, since 3\%-5\% of mRNAs remain associated with polyribosomes when cap-dependent translation initiation is inhibited in poliovirus-infected cells (Johannes et al. 1999) and a further 3\% were found to be present on the polysomes during mitosis (Qin and Sarnow 2004). This implies that internal initiation is an important cellular mechanism for regulating translation and not just a specialized viral strategy. 
Despite the large number of mRNAs that use internal ribosome entry to initiate translation, very little is known about the structures of IRESs or the ITAFs that aid ribosome recruitment. Therefore one of the major challenges in this area of research is to find common structural or sequence motifs and groups of proteins that allow coordinated regulation of subsets of cellular IRESs. Secondary structural models have been derived for a small number of cellular IRESs to date (Le Quesne et al. 2001; Pedersen et al. 2002; Bonnal et al. 2003; Mitchell et al. 2003; Yaman et al. 2003; Jopling et al. 2004; Pickering et al. 2004), although no obvious common structural features have been found, even when the IRESs of the closely related genes c-myc and L-myc are compared (Le Quesne et al. 2001; Jopling et al. 2004).

We have studied the mechanism of translation initiation of the c-myc (Le Quesne et al. 2001), Apaf-1 (Mitchell et al. 2003), L-myc (Jopling et al. 2004), BAG-1 (Pickering et al. 2004), and N-myc (Jopling and Willis 2001) IRESs and found that they all use the "land and scan mechanism" of translation initiation in which the ribosome is recruited to a site upstream of the start codon; they are therefore similar in this regard to type I picorna and entero viruses (Belsham and Jackson 2000). However, type I viral IRESs direct the ribosome to an upstream AUG codon, whereas for cellular IRESs no defined ribosome-landing codon has been identified (Le Quesne et al. 2001; Mitchell et al. 2003; Jopling et al. 2004). In picornaviruses, ribosome recruitment occurs at an AUG triplet located at the 3' end of the IRES, and this codon is $\sim 25-30$ nucleotides ( $\mathrm{nt}$ ) downstream from the start of a polypyrimidine-rich tract (Belsham and Jackson 2000). The polypyrimidine tract-binding protein (PTB or hnRNPI) has been shown to interact with, and in some cases be required for, translation initiation mediated by IRESs found in certain picornaviruses, including FMDV (Niepmann 1996), EMCV (Kaminski and Jackson 1998; Kaminski et al. 1995), poliovirus (Hellen et al. 1994), HAV (Schultz et al. 1996), and HCV (Ali and Siddiqui 1995; Anwar et al. 2000).

PTB was originally identified as a component of splicing complexes (Gil et al. 1991) and binds to polypyrimidine-rich sequences (Singh et al. 1995). It has also been implicated in polyadenylation (Lou et al. 1999), RNA stability (Tillmar and Welsh 2004; Wollerton et al. 2004), and RNA localization (Cote et al. 1999). Although the mechanisms are not always completely understood, PTB seems to act as a repressor in most of these cases by binding to pyrimidine-rich elements in the RNA and preventing the proper interaction of other (positive and negative) regulatory factors. It is in relation to IRES function that there is most evidence for a truly positive role for PTB. There are four human variants of PTB: PTB-1, PTB-2, PTB-4 (Wagner and Garcia-Blanco 2001), and $\mathrm{nPTB}$, an isoform that shows increased expression in neuronal tissues (Markovtsov et al. 2000; Polydorides et al. 2000). All isoforms of PTB are modular proteins that contain four RNA recognition motifs (RRMs) (Conte et al. 2000). However, these motifs are noncanonical (Kenan et al. 1991). The two C-terminal RRMs were thought to contain the most critical RNA-binding determinants (Conte et al. 2000), and it was suggested that RRM2 is required for PTB oligomerization (Perez et al. 1997b). However, recent data have shown that all four RRMs interact with the RNA, and that PTB exists as a monomer in solution with an elongated structure consistent with a linear arrangement of the RRMs (Simpson et al. 2004). Using SELEX, the optimal binding site for PTB-1 has been defined as 5'-CAGCCUGGUGCCUCU CUUUCGG-3' (Singh et al. 1995), but other studies have shown that the PTB recognition sequence consists of UCUU or UCUUC within a pyrimidine-rich sequence (Perez et al. 1997a). However, the dissociation constants for the interaction between PTB and these relatively short sequences are high, raising doubts over the physiological relevance of these binding sites. Indeed, the dissociation constants for the interaction of PTB with longer physiological target RNAs, such as sequence elements involved in splicing reactions or viral IRESs, are much lower (Perez et al. 1997a; Conte et al. 2000).

We showed previously that PTB is also required for the activity of certain cellular IRESs. The best characterized of these is the Apaf-1 IRES, which contains three polypyrimidine tracts and thus three potential PTB-binding sites (Mitchell et al. 2001, 2003). Two of these, which include the motif CCU, are required for IRES-mediated translation initiation, since deletion or mutation of either one of these sites reduces Apaf-1-IRES function (Mitchell et al. 2003). More recently we showed that PTB-1 also binds to the Bag-1 IRES (Pickering et al. 2003, 2004), and our data strongly suggest that PTB-1 is an essential part of the preinitiation complex since mutations that alter the PTB-binding region ablate IRES activity (Pickering et al. 2004).

Here we have generated artificial IRESs containing repeats of the CCU motif and show that when present in a stem loop this sequence is sufficient to mediate PTBdependent internal ribosome entry both in vitro and in vivo. The cellular $5^{\prime}$ UTRs tested which harbor this sequence permit PTB-dependent internal ribosome entry. Taken together these data suggest that this motif is sufficient to allow ribosome recruitment and that PTB, either alone or in conjunction with its binding partners, is directly involved in ribosome recruitment.

\section{Results}

Identification of a polypyrimidine motif present in the 5' UTRs of certain cellular mRNAs

Our mutational studies of the Apaf-1 IRES demonstrated that PTB-1 interacts with pyrimidine-rich sequences that contain CCU (Mitchell et al. 2003), a motif that varies considerably from the binding site identified by SELEX (Singh et al. 1995; Perez et al. 1997a) and could perhaps represent a cellular-IRES-specific PTB-binding region. To investigate how this motif may function in ribosome recruitment, a series of artificial IRESs (AIRESs) was created (Fig. 1A). These AIRESs were based on the polypyrimidine-rich sequences present in the 
Mitchell et al.
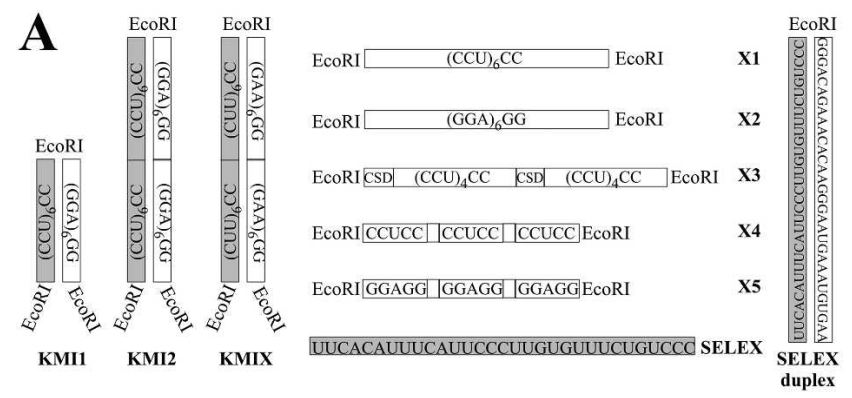

B ${ }_{\text {PRF }}$

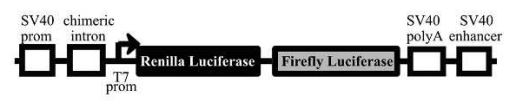

pR(AIRES)F
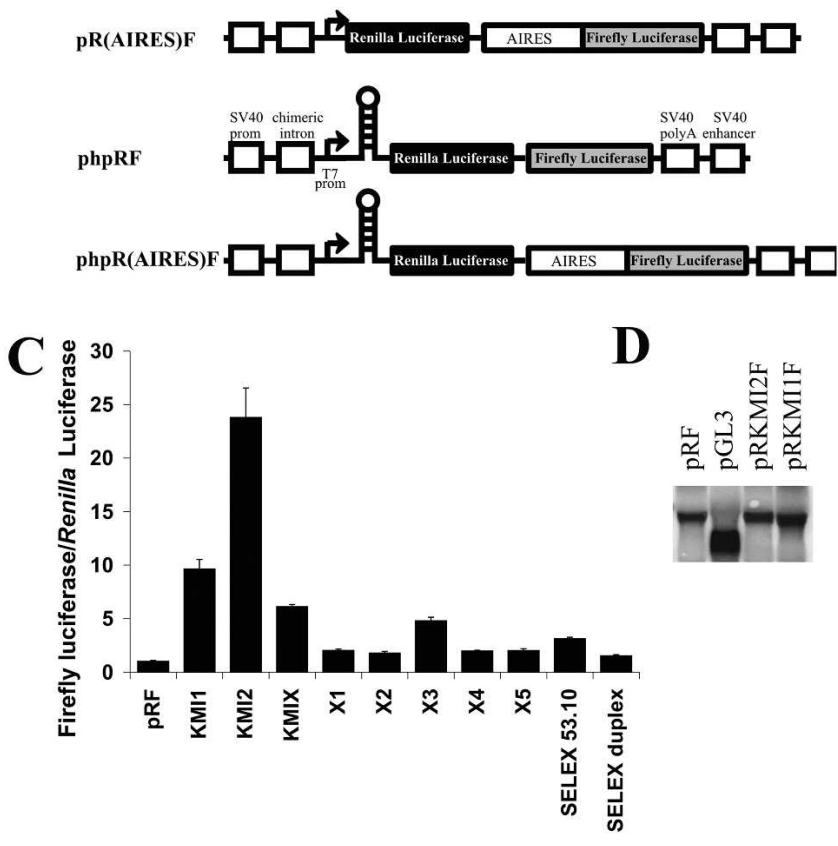

E(i)

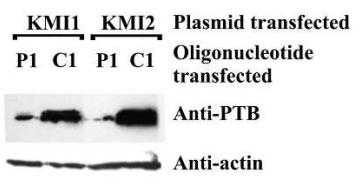

(ii)

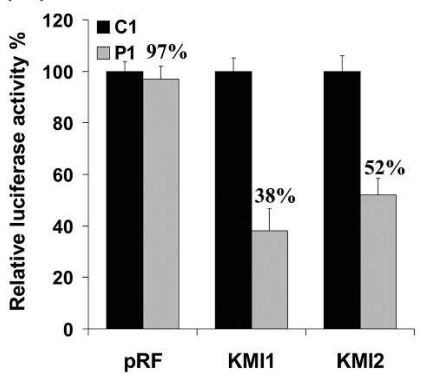

$\mathbf{F}$

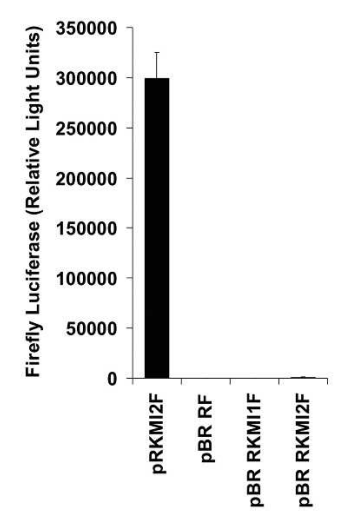

G

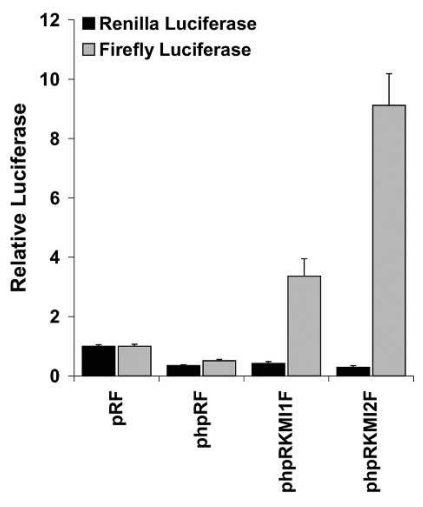

Figure 1. Generation of artificial IRESs that function in vivo and in vitro. (A) AIRESs were based on concatenated PTB-binding sites in a number of contexts, and were made by cloning synthetic DNA oligonucleotides into the intercistronic region of the dicistronic luciferase reporter plasmid pRF. The sequences of the AIRES elements are shown. $(B)$ Schematic view of the reporter construct pRF, the artificial IRES-containing construct pR(AIRES)F, and the same constructs with a hairpin inserted upstream of the Renilla luciferase cistron. $(C)$ These constructs were transfected into HeLa cells and luciferase expression determined. Expression of the downstream luciferase cistron above background (readthrough) levels is indicative of IRES activity for KMI1 and KMI2, and these show similar levels of activity to cellular IRESs. (D) HeLa cells were transfected with pGL3, pRF, pRKMI1F, and pRKMI2F, after which poly A+ was isolated from these cells. The firefly luciferase gene was used to make a radiolabeled probe and hybridized to the nylon filter. The Northern analysis of these constructs shows that the messages are full length. (E, panel i) The pRKMI1F and pRKMI2F constructs were transfected into HeLa cells where the level of PTB was reduced by RNAi. (Panel ii) The activity of these IRESs is decreased, showing that these sequences are dependent on PTB for function in vivo. $(F)$ The KMI1 and KMI2 sequences were subcloned into the dicistronic promoterless vector pBRRF. These vectors were transfected into HeLa cells; firefly luciferase produced is seen to be negligible compared with the control construct containing a promoter, suggesting that these sequences do not contain cryptic promoters. $(G)$ The KMI1 and KMI2 sequences were subcloned into phpRF, containing a stable hairpin upstream of the Renilla cistron that impedes ribosome scanning. These vectors were transfected into HeLa cells. Values presented are normalized against the levels of luciferase produced from the plasmid phpRF. No effect on AIRES function was observed.

Apaf-1 IRES, and a number of different sequences were tested which are predicted to form either double- or single-stranded regions of RNA (Fig. 1A). AIRESs with mutations in the CCU motif or in the reciprocal sequence were also generated. In addition, as a control, the PTB binding sequence as identified by SELEX (Singh et al. 1995) was also used either in single-stranded form or as part of a hairpin (Fig. 1A). These sequences were inserted between the cistrons of pRF (Fig. 1B; Stoneley et al. 2000b) to create a series of plasmids (pRKMI1F, pRKMI2F, pRKMIXF, pRX1F, pRX2F, pRX3F, pRX4F, and pRSELEXF). These constructs were transfected into HeLa cells, and the luciferase activities were determined (Fig. 1C). 
The only sequences that showed appreciable IRES activity were those in which the polypyrimidine-rich motif containing repeats of CCU was present as a part of a predicted stem-loop structure. Thus KMI1 and KMI2 sequences were sufficient to allow internal ribosome entry, and KMI2, which contains more repeats of the CCU motif, was twice as active as KMI1 (Fig. 1C). KMIX, in which the motif has been mutated to $(\mathrm{CUU})_{\mathrm{n}}$, was not found to be as active as KMI2, which it closely resembles, thus providing evidence that it is the $(\mathrm{CCU})_{\mathrm{n}}$ motif which is important for the activity of an AIRES rather than the overall polypyrimidine component (Fig. 1C). Other AIRESs based on the KMI2 construct were also produced into which mutations were introduced in order to produce mismatches and thus hairpin constructs with less stability. We observed that the hairpin structure is able to tolerate up to 12 base mismatches in the 50-nt (GGA) half of the hairpin without significant loss of activity, as long as the $(\mathrm{CCU})_{\mathrm{n}}$ sequence is not altered (data not shown). This is not entirely unexpected since the hairpins seen in cellular IRESs are seldom perfect and often contain several mismatches and bulges. Finally, we also examined the ability of a previously determined PTB binding sequence (SELEX 53.10) to act as an IRES, either as a single-stranded RNA (ssRNA) sequence or as part of a duplex structure. However, we have not been able to show that the SELEX sequence is able to act as an IRES in either form (Fig. 1C).

To test the effect of PTB-1 expression on IRES function in vivo, PTB-1 levels were reduced in HeLa cells by using RNA interference (RNAi) technology. Cells were transfected with an siRNA duplex (P1) known to be effective in reducing levels of PTB-1 or control siRNAs (C1) as described previously (Wollerton et al. 2004), and then after $24 \mathrm{~h}$ the cells were transfected with the dicistronic reporter constructs $\mathrm{pRKMI} 1 \mathrm{~F}$ and $\mathrm{pRKMI} 2 \mathrm{~F}$. After a further $48 \mathrm{~h}$, cells were harvested, and lysates were separated by SDS-PAGE, immunoblotted, and probed with anti-PTB-1 antibodies (Fig. 1E, panel i). The same samples were then assayed to monitor luciferase expression (Fig. 1E, panel ii). The PTB-1 levels were reduced to $<10 \%$ of the control values using RNAi, in agreement with previously published work using the same target sequences (Wollerton et al. 2004). The control siRNA had no effect on firefly luciferase produced from the dicistronic constructs. However, in cells with reduced levels of PTB, the IRES-dependent luciferase levels were reduced to $38 \%$ in the case of KMI1 and $52 \%$ with KMI 2 (Fig. 1E, panel ii).

Northern analysis was performed on RNA isolated from cells transfected with pRKMI1F and pRKMI2F to ensure that KMI1 and KMI2 function as IRESs and do not contain either promoter elements or splicing elements (Fig. 1D). Only one band of the correct size was present in each case, indicating that the constructs produced full-length dicistronic RNAs. RNA derived from cells transfected with pGL3 (Stoneley et al. 2000a) as a control produced a much smaller monocistronic band on the same gel (Fig. 1D). As further proof that KMI1 and KMI2 do not contain cryptic promoters, the sequences were inserted into the dicistronic promoterless construct pBRRF and transfected into HeLa cells. No significant luciferase activity was detected in cell lysates, confirming that these sequences do not act as promoters (Fig. 1F). In addition, DNA encoding KMI1 and KMI2 was introduced into the hairpin-containing construct phpRF (Fig. 1B; Coldwell et al. 2001). The presence of the hairpin did not affect the expression of firefly luciferase, implying that KMI1 and KMI2 do not stimulate readthrough (Fig. 1G). Collectively these data indicate that KMIl and KMI2 can promote internal initiation in vivo in a PTBdependent manner.

\section{PTB binds preferentially to double-stranded RNA (dsRNA)}

KMI1 and KMI2 are palindromic sequences, and hence are predicted to form RNA stem-loop structures. To confirm that these sequences do adopt a hairpin structure, we determined the accessibility of residues in KMI1 and KMI2 to RNAse V1 (cleaves dsRNA) and chemical modifying agents (DMS, kethoxal, CMCT, as described previously; Supplementary Data A). These data were used to constrain the Mfold algorithm, and thereby derive structural models for KMI1 and KMI2 (Fig. 2A, panels i,ii). As predicted, the structures formed by these sequences are stem loops, with the PTB-binding motif present in the stem. Further structural studies were performed on KMI1 and KMI2 in the presence of PTB. This analysis revealed that $\mathrm{PTB}$ inhibited the accessibility of residues in KMI1 and KMI2 to RNAse V1, but there was no concomitant increase in the chemical modification at these sites (data not shown), indicating that the RNA structure is not being opened up. Hence PTB appears to interact with the unaltered stem region of the AIRESs.

Experiments were then performed to test whether PTB was interacting with dsRNA or ssRNA. Radiolabeled KMI1 and KMI2 RNAs were generated by in vitro transcription, and these were used in electrophoretic mobility shift assays (EMSAs). PTB-1, PTB-2, and $\mathrm{nPTB}$ all interacted strongly with KMI1 and KMI2, as all the RNA was retarded in the presence of these proteins, while a weaker interaction was observed with PTB-4 (Fig. 2B, panels i,ii). Neither unr nor La bound to these sequences (Fig. 2B, panels i,ii).

Radiolabeled oligonucleotides were then generated that corresponded to each side of the stem of the KMI1 and KMI2 hairpin structures. After denaturation the combined oligonucleotides were annealed to allow the formation of RNA helix. The interaction of PTB with the double-stranded sequences was then analyzed using EMSAs (Fig. 2B, panels iii). In each case $\sim 50 \%$ of the material formed dsRNA. Upon the addition of PTB-1, a retarded band was detected showing that PTB bound to the RNA. Moreover these RNA-protein complexes had approximately the same mobility as the complexes formed between PTB-1 and KMI1 or KMI2 (Fig. 2B, panel iii). Crucially there was a corresponding decrease in the amount of radiolabeled double-stranded oligonucleotide at the bottom of the gel, but no decrease in the amount 
Mitchell et al.
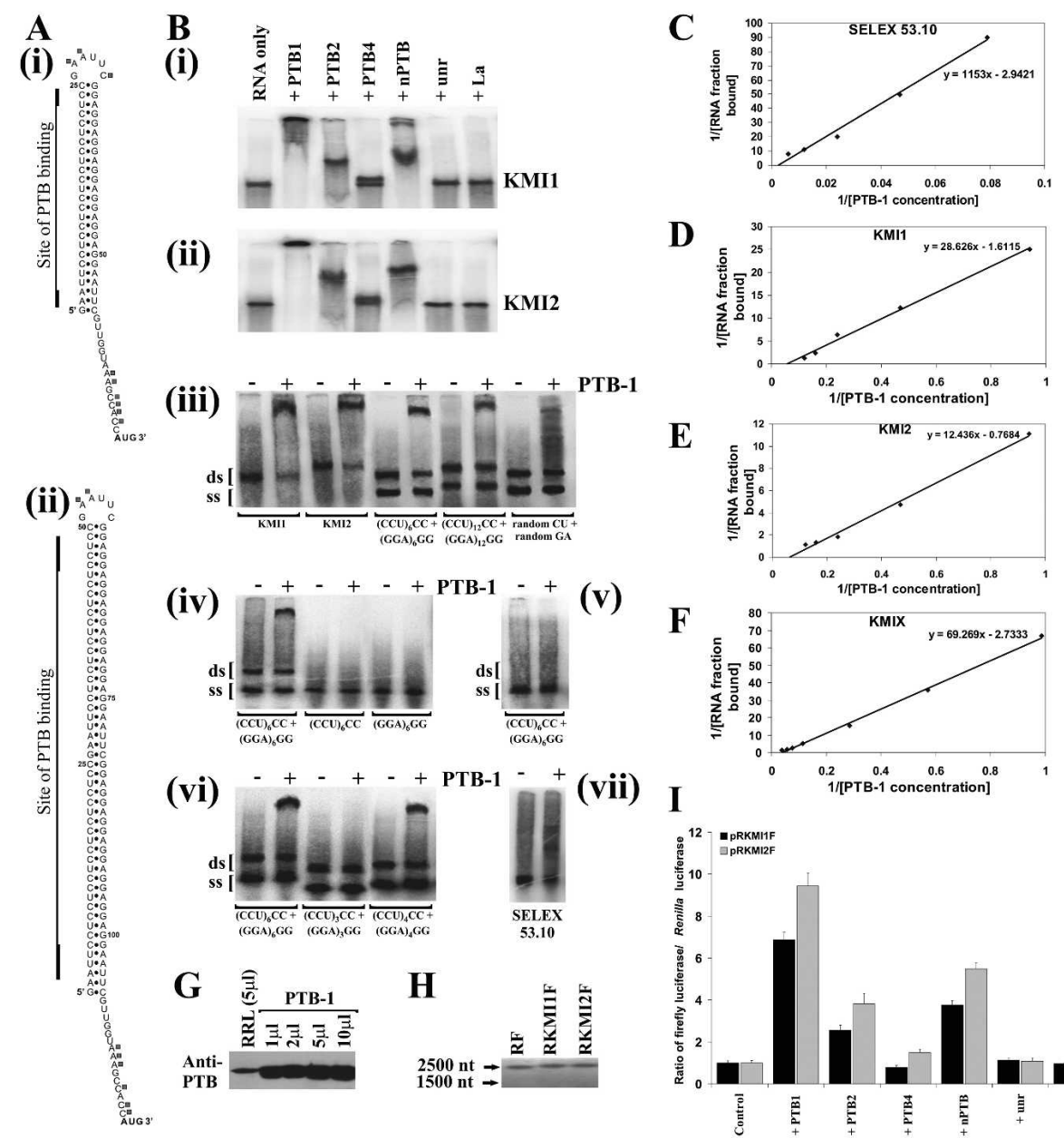

$\mathbf{E}$
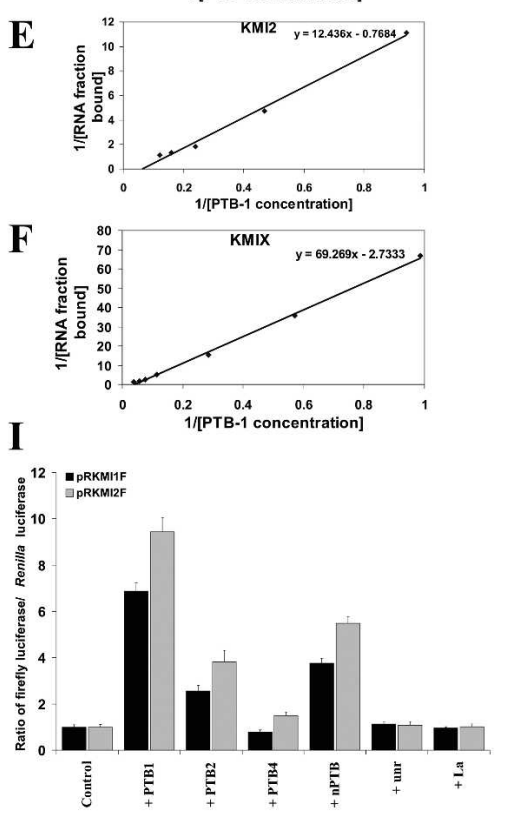

Figure 2. PTB binds to dsRNA. (A) Structural models for KMI1 (panel i) and KMI2 (panel ii) derived from chemical and enzymatic mapping (Supplementary Data A). The structures formed by these sequences are double-stranded hairpins as predicted. Squares indicate residues modified by DMS. Regions of PTB binding were inferred from areas of protection from RNase V1 cleavage and are indicated. $(B$, panels i,ii) EMSAs were performed using the radiolabeled KMI1 or KMI2 RNA on TBE/acrylamide gels in the presence of PTB isoforms $(0.1 \mu \mathrm{g}$ ) or control proteins as indicated. (Panel iii) Radiolabeled ssRNA (ss) oligonucleotides comprised of the forward $\left[(\mathrm{CCU})_{6} \mathrm{CC}\right.$ or $\left.(\mathrm{CCU})_{12} \mathrm{CC}\right]$ or reverse $\left[(\mathrm{GGA})_{6} \mathrm{GG}\right.$ or $\left.(\mathrm{GGA})_{12} \mathrm{GG}\right]$ strands of $\mathrm{KMI} 1$ or KMI2 were heated to $80^{\circ} \mathrm{C}$ and allowed to cool slowly to room temperature. In each case $\sim 50 \%$ of the oligonucleotides formed dsRNA (ds) as marked. These were incubated with PTB-1 and the products of the reactions separated on TBE/acrylamide gels. These EMSAs were also repeated using a control polypyrimidine sequence that did not harbor the $(\mathrm{CCU})_{\mathrm{n}}$ motif and its corresponding complementary RNA primer pair. (Panel iv) The forward or reverse single strands generated were incubated with PTB and the products of the reactions separated on TBE/acrylamide gels; it can be seen that PTB does not act with the single-stranded sequences. (Panel $v$ ) In this case the radiolabeled oligonucleotides were heated to $80^{\circ} \mathrm{C}$ and then snap frozen; no dsRNA is formed and no PTB interaction is observed. (Panel vi) To define the minimum number of repeats of CCU that PTB could bind, dsRNAs were generated that contained three and four repeats of the motif. (Panel vii) The data show that PTB can bind to four but not to three repeats. EMSAs were also repeated using the SELEX PTB binding sequence, and the data suggest that PTB binds poorly to this sequence. $(C)$ EMSAs were performed using various concentrations $(1.4-10 \mu M)$ of PTB and the bound fraction of RNA determined using a personal FX PhosphorImager (Bio-Rad). A graph of the reciprocals of bound RNA against protein concentrations produces a straight line trend for SELEX binding, but as shown on the graph $(y=1153 x)$, the dissociation constant $\left(\mathrm{K}_{\mathrm{d}}\right)$ is quite poor. $(D)$ The affinity of binding of PTB1 to KMI1 $(y=28.6 x)$ is $\sim 40$-fold stronger than binding to SELEX RNA. $(E)$ Binding of PTB to KMI2. In this case the dissociation constant $(y=12.4 x)$ is $\sim 80$-fold better than that seen with the SELEX sequence. (F) PTB is able to bind to KMIX, as expected, but with a much lower affinity of binding than to KMI2 (y=69.3x). However, the dissociation constant is still better than that seen for the SELEX sequence. $(G)$ Immunoblot of rabbit reticulocyte lysates with the addition of purified PTB demonstrating that the PTB levels were increased by approximately fivefold when only $0.1 \mu \mathrm{g}$ protein was used. $(H)$ Radiolabeled dicistronic RNAs derived from pRF, pRKMI1F, and pRKMI2F were incubated in rabbit reticulocyte lysates in the presence of PTB. The RNAs were then extracted and run on a TBE/agarose gel before exposure on a PhosphorImager plate. This shows that these RNAs remain intact during the assay period. (I) Rabbit reticulocyte lysates were primed with capped dicistronic RNA derived from the plasmid pRKMI $(1 / 2) \mathrm{F} \pm$ the addition of $200 \mathrm{ng}$ of PTB isoforms shown. The firefly:Renilla ratios are expressed relative to that of the control plasmid pRF, which was assigned a value of 1 . 
of single-stranded oligonucleotides (Fig. 2B, panel iii), suggesting that PTB is indeed binding dsRNA. As a control, annealed oligonucleotide pairs of the same length but containing a random polypyrimidine sequence were used and were found to interact with PTB-1 inefficiently (Fig. 2B, panel iii). To provide further evidence that PTB interacts with these dsRNA, the individual oligonucleotides were incubated with PTB-1 (Fig. 2B, panel iv). In addition we assessed the interaction between PTB and complementary oligonucleotide pairs that had been denatured and then rapidly cooled so that little or no dsRNA was generated (Fig. 2B, panel v). In both cases, upon the addition of PTB-1 no retarded band was observed, suggesting that PTB-1 interacts poorly with these ssRNA sequences (Fig. 2, panels iv,v). To define the minimum number of repeats of CCU to which $\mathrm{PTB}$ could bind, dsRNA oligonucleotides consisting of either three, four, or six repeats of this motif were used in EMSAs (Fig. 2B, panel vi). The data show that PTB is unable to interact with three repeats of this motif but can bind to four repeats (Fig. 2B, panel vi), which allows us to estimate the minimum length of RNA required for PTB binding. It was shown previously that PTB-1 interacts with the ssRNA sequence as defined by SELEX (Singh et al. 1995); therefore EMSAs were also performed with this sequence as a comparison. Although a shifted band was observed with this sequence, PTB-1 bound much less strongly to the SELEX oligonucleotide than to KMI1 or KMI2 (Fig. 2B, panel vii). Further EMSAs were performed to deduce the dissociation constants $\left(\mathrm{K}_{\mathrm{d}}\right)$ for the binding of PTB to KMI1, KMI2, KMIX, and SELEX RNA. The fraction of bound RNA was assessed using a PhosphorImager, and was plotted on a graph relative to the amount of protein added to the reaction (Black et al. 1998). From the graphs, the $K_{d}$ for these interactions can be calculated, and we are able to show that while PTB does indeed bind to the SELEX sequence (Fig. 2C), it binds with much higher affinity to KMI1 (40-fold higher) (Fig. 2D) and to KMI2 (90-fold higher) (Fig. 2E). It can also be seen that PTB is able to bind to KMIX, less strongly than to KMI2, but more strongly than to the SELEX sequence (Fig. 2F). Since PTB binds to polypyrimidine tracts, it would be expected to bind to a sequence comprised of $(\mathrm{CUU})_{\mathrm{n}}$ i however, the binding affinity is approximately sixfold lower than the binding to KMI2, a result which further emphasizes the importance of the specific motif CCU.

To investigate the dependence of the AIRESs on PTB in vitro, RNA was generated from pRKMIIF and pRKMI2F and used to prime rabbit reticulocyte lysates. Western analysis was performed to assess the levels of PTB in such lysates. It can be seen that the amount of PTB protein added to these reactions increases the endogenous level by approximately fivefold, as determined by densitometry (Fig. 2G). In addition the RNA used in these reactions was analyzed, and the data show that this remains intact during the assay period (Fig. $2 \mathrm{H}$ ). In the absence of PTB, KMI1 and KMI2 displayed little IRES activity in vitro (Fig. 2I). However when the translation reactions were supplemented with PTB-1, KMI1 and
KMI2 stimulated downstream cistron expression by sevenfold and 10-fold respectively. The other PTB isoforms also activated the AIRESs, albeit to a lesser degree (Fig. 2I). It should also be noted that in the presence of PTB-1 these artificial IRESs are far more active than other cellular IRESs that we have tested to date in rabbit reticulocyte lysates.

\section{Analysis of cellular mRNAs that harbor the specific PTB binding sequence}

To test the prevalence of this motif, a database of $5^{\prime}$ UTRs, the UTRdb (Pesole et al. 2002), was searched, using MEME and MAST (Bailey and Gribshaw 1998) for mRNAs that contained three or more repeats of the Apaf-1 PTB-binding motif, CCUCC, within a polypyrimidine-rich region. Approximately 3000 mRNAs met these criteria, which amounts to $\sim 10 \%$ of the database (data not shown). In contrast when the database was screened for mRNAs that contained three or more repeats of UCUUC, only $1.2 \%$ of the $5^{\prime}$ UTRs were found to contain this sequence. Based on this analysis, the core PTB-binding motif $(\mathrm{CCU})_{\mathrm{n}}$ could potentially participate in internal initiation in a very large number of cellular mRNAs, and as such would represent the first common functional motif to be identified in cellular IRESs. Examination of the mRNAs identified by the screen revealed a preponderance of genes whose products are important in the control of cell growth or cell death (data not shown).

Six of the mRNAs identified in the screen (GTF2, Mnt, Myb, MTG8, ET4F, and BTEB2) were studied in more detail to determine whether their translation was mediated by internal ribosome entry. Thus the cDNA of the 5' UTRs from these genes were subcloned into the intergenic region of the dicistronic reporter vector $\mathrm{pRF}$ (Stoneley et al. 2000b). HeLa cells were transfected with the resulting constructs, and the lysates were assayed for luciferase activity. All of those tested were able to efficiently direct translation from the downstream cistron, albeit with different degrees of efficiency (Fig. 3A). The 5' UTRs of Mnt (which was shown previously to contain an IRES) (Stoneley et al. 2001), c-myb, and MTG8a were selected for further analysis, and a number of controls were carried out to rule out alternative mechanisms that could in theory mimic internal ribosome entry. Possible mechanisms are enhanced ribosomal reinitiation at the firefly luciferase initiation codon and the production of functional firefly luciferase mRNAs due to splicing, RNA cleavage, or a cryptic promoter. Thus the 5' UTRs of Mnt, c-myb, and MTG8a were subcloned into the dicistronic vector phpRF, which contains a stable hairpin upstream of the Renilla luciferase coding region to impede scanning. The presence of the artificial hairpins did not reduce firefly luciferase expression, indicating that increased readthrough could not account for the activity observed (Fig. 3B). Northern analysis was performed to test whether the dicistronic mRNAs were intact (Fig. 3C); however, no monocistronic firefly luciferase mRNAs were detected that could account for the enzyme 
Mitchell et al.
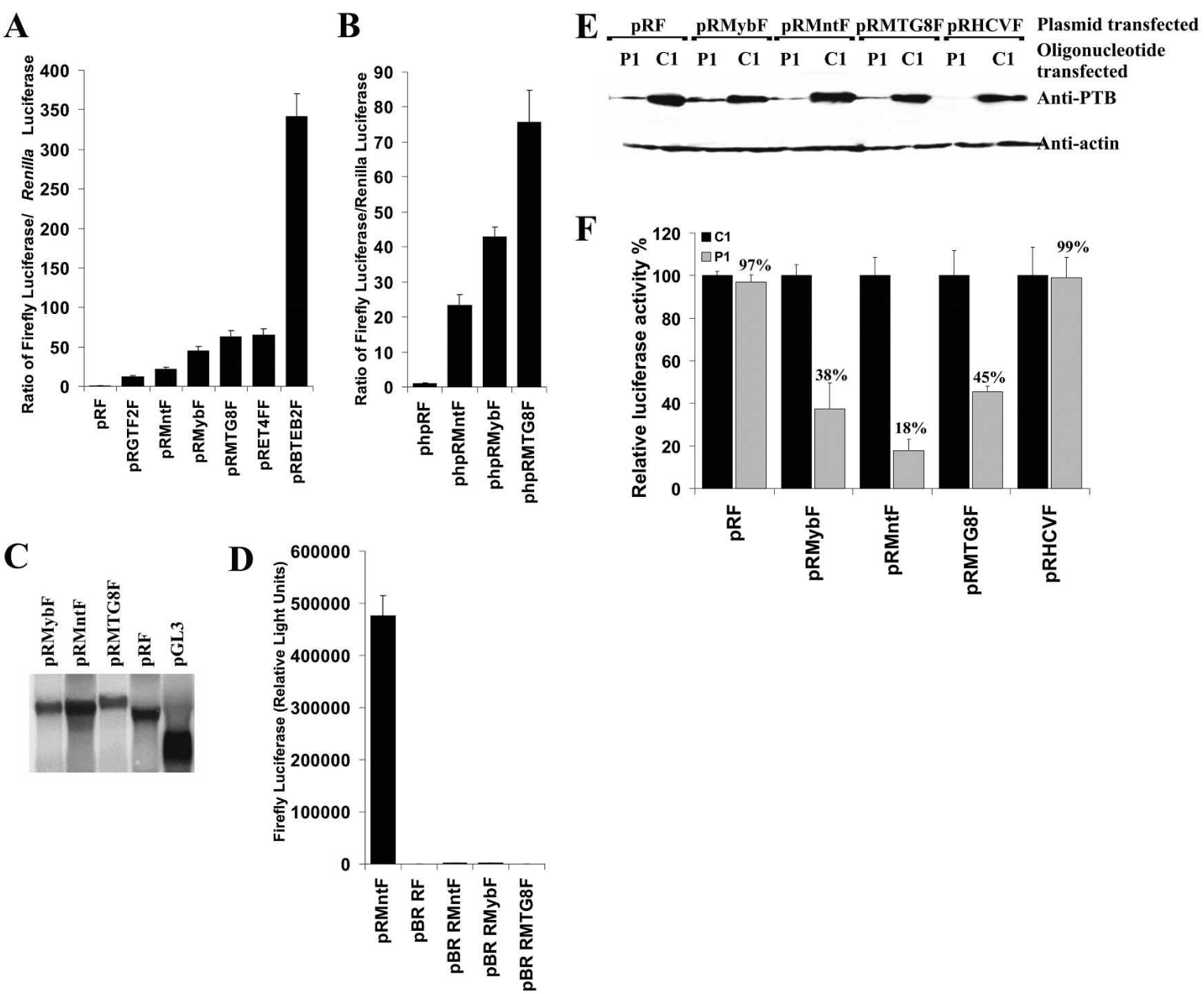

Figure 3. Cellular 5' UTRs that harbor the CCU motif contain IRESs. (A) The 5' UTRs of GTF2, Mnt, Myb, MTG8a, ET4F, and BTEB2 were obtained by RT-PCR and inserted into pRF in-frame with firefly luciferase, before transfection into HeLa cells. Values presented are normalized against the levels of luciferase produced from the control plasmid pRF. IRES activity is comparable with previously published IRESs. (B) The 5' UTRs of Mnt, Myb, and MTG8a were subcloned into phpRF and transfected into HeLa cells. Values presented are normalized to the control plasmid phpRF. No reduction of firefly luciferase activity is observed in this vector, implying that this activity is not due to readthrough. $(C)$ HeLa cells were transfected with pGL3, pRF, pRMntF, pRMybF, or pRMTG8aF, and poly A+ RNA was isolated from these cells. Northern blotting was performed as described previously (West et al. 1995), and the resultant data show that the constructs are full-length; i.e. no internal cleavage or splicing is occurring. (D) The 5' UTRs of Mnt, Myb, and MTG8a were subcloned into the dicistronic promoterless vector pBRRF. These vectors were transfected into HeLa cells where the amount of firefly luciferase produced is $\sim 500$-fold less than from the control construct which contains a promoter, indicating that these sequences do not contain cryptic promoter sequences. $(E)$ RNAi was used to reduce the expression of PTB-1 in HeLa cells to $10 \%$ of the level in untreated cells, as judged by Western analysis. $(F)$ The cells were then transfected with the dicistronic plasmids shown which, with the exception of that containing the HCV IRES, all show a reduction in activity of the IRES-mediated translation.

activity detected. PGL3 is a vector encoding firefly luciferase alone and was therefore included to indicate the minimum size of functional firefly luciferase-encoding mRNAs. Finally, the regions of DNA encoding the $5^{\prime}$ UTRs of Mnt, c-myb, and MTG8a were inserted into a dicistronic promoterless construct (pBRRF), and no significant luciferase activity was detected, showing that these sequences do not harbor cryptic promoter sites (Fig. 3D). Taken together, these data strongly suggest that the 5' UTRs of these three mRNAs identified in the screen are capable of initiating translation by internal ribosome entry.

To test the effect of PTB-1 expression on IRES function in vivo, PTB-1 levels were reduced in HeLa cells by using RNAi with the dicistronic reporter constructs shown (Fig. 3E,F). Treated cells were harvested and lysates were separated by SDS-PAGE, immunoblotted and probed with anti-PTB-1 antibodies (Fig. 3E). The same samples were then assayed to monitor luciferase expression (Fig. 3F). In cells with reduced levels of PTB, the IRES-dependent luciferase levels were reduced to $38 \%$ in the case of the c-myb IRES, $45 \%$ with the MTG8a IRES, and $18 \%$ with the Mnt IRES (Fig. 3F). The HCV IRES was also tested in this system, and the data show that reducing PTB-1 levels had no effect on the activity of this IRES (Fig. 3F). This would suggest that PTB-1 is not essential 
for HCV IRES function, even though this protein has been shown to interact with HCV in vitro (Ali and Siddiqui 1995; Anwar et al. 2000) and HCV IRES activity can be stimulated by PTB in vivo (Gosert et al. 2000). Moreover, these data imply that PTB-1 is a critical component of the ITAF complex that is required for certain cellular IRESs to function.

c-myb, Mnt, and MTG8a IRESs require PTB for function in vitro and in vivo

To test the ability of the IRESs located in MTG8a, Mnt, and $c-m y b$ to bind to PTB isoforms, EMSAs were performed. The three IRESs were subcloned into the vector pSKL, which was then linearized with NcoI, and radiolabeled RNA was generated by in vitro transcription. The RNAs were incubated with the proteins shown and the reaction products separated on 5\% TBE acrylamide gels (Fig. 4A [myb], C [mnt], E [MTG8]). All three IRESs interact with PTB isoforms, but there are differences in these interactions. Thus, they all bind PTB-1 and PTB-2; however, only the c-myb IRES binds strongly to PTB-4
(Fig. 4A). In contrast, MTG8a and Mnt IRES RNA, but not c-myb IRES RNA bind to nPTB (Fig. 4C,E). Similar results were obtained with UV cross-linking analysis (data not shown). As controls, three additional RNAbinding proteins (La, DAP5, and ITAF45) were also incubated with the three IRESs. None of these had an effect on RNA mobility, suggesting that the interactions with the PTB isoforms were specific. To assess the effects of PTB isoforms on IRES-mediated translation initiation in vitro, reticulocyte lysates were primed with in vitro transcribed RNA derived from $\mathrm{pRF}, \mathrm{pRMybF}, \mathrm{pRMntF}$, and $\mathrm{pRMG} 8 \mathrm{~F}$ in the presence or absence of $\mathrm{PTB}$ proteins (Fig. 4B,D,F). Luciferase assays were performed, and the activity of each IRES is shown relative to that produced by $\mathrm{pRF}$ in the absence of protein. An increase in IRES function in vitro was observed with the PTB isoforms that bind most strongly to the IRES. For example, translation initiation mediated by the MTG8a IRES was stimulated by the presence of PTB-1, PTB-2, and $\mathrm{nPTB}$ but not PTB-4 (Fig. 4F).

To test whether the $(\mathrm{CCU})_{\mathrm{n}}$ repeated motif could affect the function of these IRESs in vitro, RNAs encoding
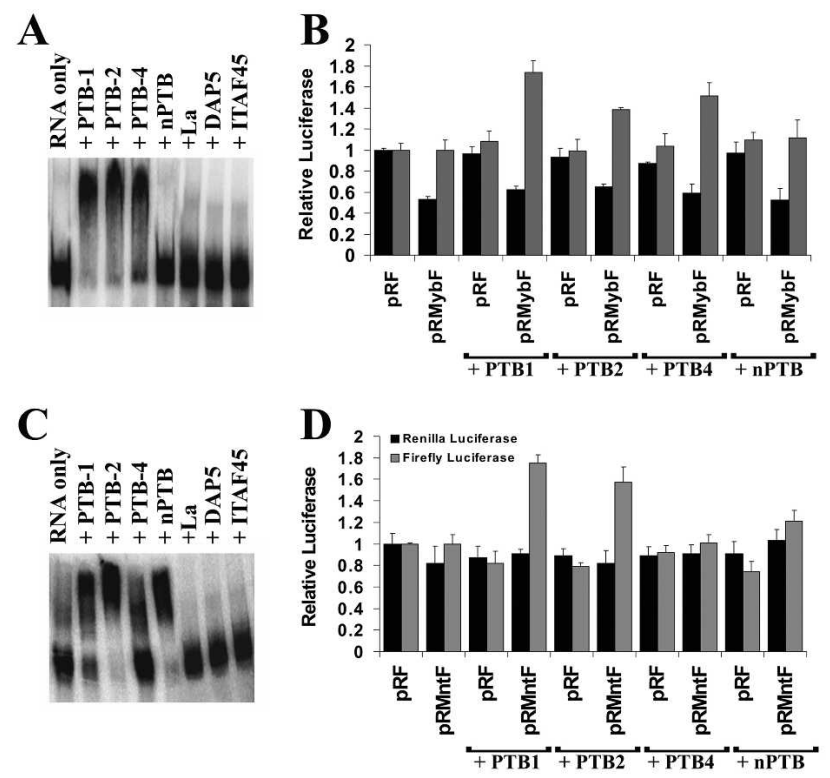
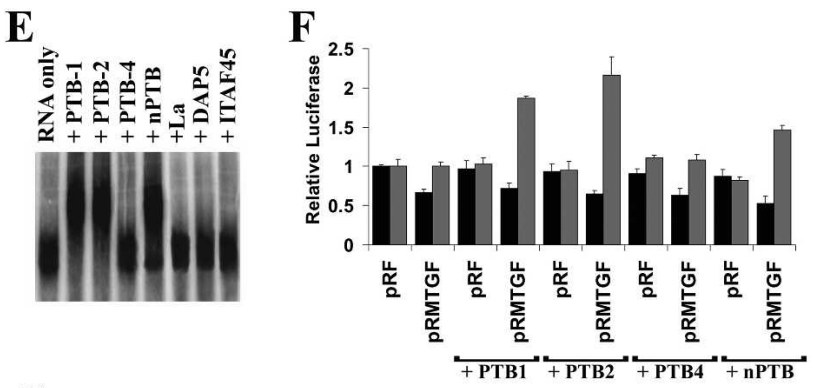

G

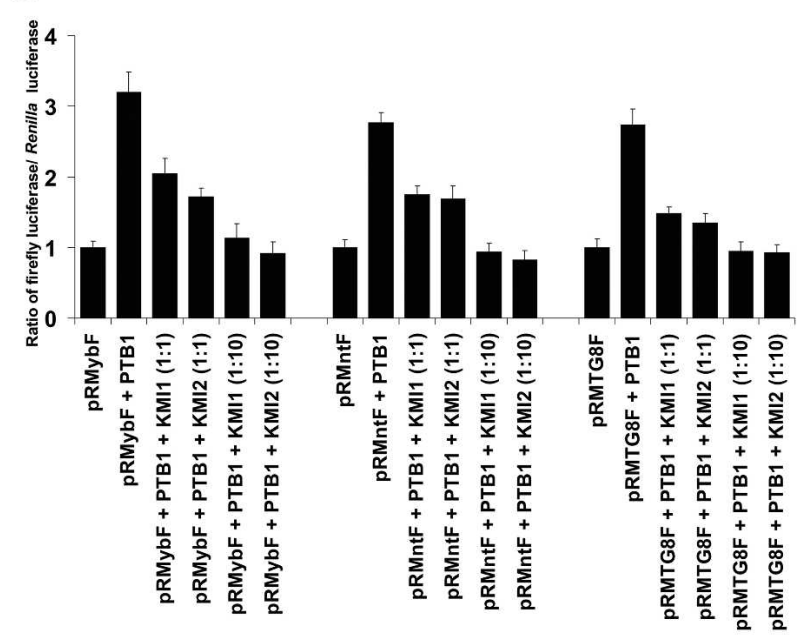

Figure 4. $(\mathrm{CCU})_{\mathrm{n}}$-containing IRESs require PTB for function in vitro and in vivo. $(A, C, E)$ EMSAs were performed using the radiolabeled IRES RNA (Myb $[A]$; Mnt [C]; MTG8a [E]) on TBE/acrylamide gels in the presence of one of four PTB isoforms (0.1 $\mu$ ) or control proteins as indicated. $(B, D, F)$ Rabbit reticulocyte lysates were primed with capped dicistronic RNAs derived from the plasmid pRUTRF (as shown) \pm the addition of $200 \mathrm{ng}$ of the PTB isoforms shown. The firefly and Renilla values are expressed relative to that of the control plasmid pRF, which was assigned a value of 1 . All experiments were performed in triplicate on at least three independent occasions. (G) Rabbit reticulocyte lysates were primed with capped dicistronic RNA derived from the plasmid pRUTRF as shown \pm the addition of $200 \mathrm{ng}$ of PTB-1 and a 1:1 or 1:10 molar excess of KMI1 or KMI2 RNA. It can be seen that KMI1 and KMI2 compete efficiently with cellular IRESs in vitro for PTB. 
the KMI1 and KMI2 AIRES sequences were generated and used as competitive inhibitors in in vitro translation reactions primed with dicistronic RNAs generated from pRMybF, pRMntF, and pRMTG8aF (Fig. 4G). In each case the artificial sequences competed for binding with the cellular IRESs, and a 10-fold molar excess of either artificial sequence was sufficient to inactivate the c-myb, Mnt, or MTG8a IRESs in vitro.

Derivation of structural models for the Mnt, MTG8a, and $c$-myb IRESS

To investigate how PTB was interacting with these cellular IRESs, it was first necessary to derive structural models (Supplementary Data B; see Mitchell et al. 2003). All three IRESs are highly structured, but there is no apparent similarity between the structures (Fig. 5A). In all cases the polypyrimidine-rich regions of RNA that contain the CCU motif are present in both singlestranded loop regions and in long stem structures.

Experiments were then performed to locate the site at which the ribosome enters these cellular IRESs, so that this information could be included in our models. For cellular IRESs studied thus far, the data suggest that the 40S subunit lands, and instead of initiating translation directly, scans to an authentic start codon further downstream (Le Quesne et al. 2001; Mitchell et al. 2003; Pickering et al. 2003; Jopling et al. 2004). Out-of-frame AUG codons were inserted at various positions in each IRES. If the ribosome lands upstream of one of these codons and scans through, it would be expected to initiate translation from this AUG, resulting in synthesis of a nonfunctional peptide and a corresponding decrease in firefly luciferase levels. The data obtained with the c-myb IRES indicate that it falls into the "land-and-scan" class and that ribosome entry occurs between nucleotides 9 and 66 (Fig. 5B). To test whether the effect of these mutations was due to the creation of an upstream open reading frame (ORF) within the 5' UTR and was not a consequence of change in IRES structure, the two sequences that reduced IRES function were altered to UUG. The UUG mutations had no effect on IRES function, suggesting that the structure was not altered. The Mnt and MTG8a IRESs differ from the c-myb IRES and other cellular IRESs previously studied as out-of-frame AUG codons have no effect on IRES activity, suggesting that the ribosome enters at the start codon (Fig. 5C,D).

Location of the PTB-binding site on c-myb, Mnt, and MTG8a IRESS

To determine the minimum region of each IRES that was required for function and whether these regions harbored the polypyrimidine tracts, a series of constructs containing fragments of each IRES was generated (Fig. 6A, panels i-iii). This deletion series was transfected into HeLa cells, and luciferase expression was determined to assess the affect of the truncations (Fig. 6A, panels i-iii). The data show that the $5^{\prime}$ end of the c-myb IRES is necessary for activity (Fig. 6A, panel i), whereas the middle section of the Mnt IRES has as much activity as the full-length IRES (Fig. 6A, panel ii) and the minimal active element in the MTG8a IRES resides in the $3^{\prime}$ end of the $5^{\prime}$ UTR (Fig. 6A, panel iii). The positions of the polypyrimidine tracts in each IRES are shown (shaded areas, Fig. 6B, panels $\mathrm{i}-\mathrm{iii})$, and it can be seen that the regions that display IRES activity contain polypyrimidine tracts which harbor the CCU motif (Fig. 6B). Moreover the minimal active elements for each IRES all bind PTB in EMSAs (data not shown). The correlation between PTB binding and IRES activity suggests that the interaction of PTB with these polypyrimidine-rich elements is important for internal initiation.

RNA footprinting studies were then performed to identify more precisely the region of PTB-1 binding and to determine the effect that this protein has on the structure of these three IRESs (Supplementary Data C). In the case of the c-myb IRES the data suggest that PTB- 1 interacts with the large double-stranded stem structure (Fig. 5A, panel i), since in the presence of PTB-1, RNAse VI no longer cleaves the RNA in this region. Furthermore, there was no corresponding modification of the RNA by chemicals that interact with single-stranded regions, suggesting that PTB-1 remains bound to this part of the RNA rather than promoting a structural change to a more single-stranded conformation (Supplementary Data C). PTB also caused a minor structural alteration in the loop where the ribosome is assumed to bind, with the result that this region adopts a more open conformation (Fig. 6D, panel i). On the Mnt IRES, PTB-1 binds across two stems and the single-stranded loop region between them, as these regions were protected from the action of both RNAse VI and chemical modification (Fig. 5A, panel ii). No other changes were observed in the Mnt IRES structure upon PTB binding. PTB-1 bound both a double-stranded stem and a single-stranded polypyrimidine tract that follows this stem on MTG8a IRES RNA (Fig. 5A, panel iii).

To confirm these data, a series of mutants was created for all three IRESs to alter the regions to which PTB was bound (Fig. 6C, panels i-iii). The dicistronic constructs containing these mutated IRESs were transfected into HeLa cells to test for effects on internal initiation. The data show that even small changes to the core polypyrimidine sequence are sufficient to impair IRES function, again showing that for this set of IRESs the polypyrimidine motif is critical for activity. Although we have carried out an extensive mutational analysis on these cellular IRESs, we have not yet found any other critically important regions within the minimal active element (data not shown). We also performed EMSAs on these mutants, and are able to show that those which no longer function as IRESs are also no longer able to bind PTB (data not shown).

The structures of the IRESs are shown with the PTBbinding sites marked (Fig. 6D, panels i-iii). Of the three IRESs, only the structure of the c-myb IRES was affected by PTB binding. In this case PTB caused a minor structural alteration in the loop where the ribosome binds, 


\begin{abstract}
A Myb 5'-UTR
$\begin{array}{rlr}1 & \text { ATATCAACCT GTTTCCTCCT CCTCCTTCTC CTCCTCCTCC GTGACCTCCT } & 50 \\ 51 & \text { CCTCCTCTTT CTCCTGAGAA ACTTCGCCCC AGCGGTGCG AGCGCCGCTG } & 100\end{array}$ 101 CGCAGCCGGG GAGGGACGCA GGCAGGCGGC GGGCAGCGGG AGGCGGCAGC 150 151 CCGGTGCGGT CCCCGCGGCT CTCGGCGGAG CCCCGCGCCC GCCGCGCCA 201 GGATG

\section{Mnt 5'-UTR}

1 TTATATTTTG CAAATATTTT GAGAGACATT GATTTTTCTC CCCGTGCTCC 50 1 CCCGTTCTTC CCTGCGGAGT GCGCTGCGCC GCCCAGCCCT GTCGCCCCCC 100 101 GGAGGTGATC CCTCCCTCCT GCCTGCCCGC GAGCCTGACC TGTGCCCGGC 150 $\begin{array}{ll}151 & \text { TCGCGGGCCG CAGCCTCGGC CCCGGCGCGC CCCCGGCAGC ICTCGGCGCG } 200 \\ 201 \text { ATG } & \end{array}$
\end{abstract}

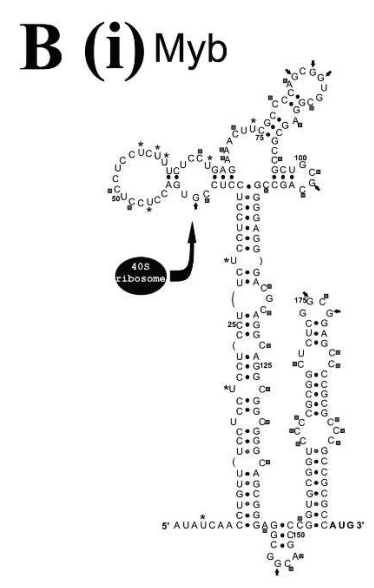

(ii) $\mathrm{Mnt}$
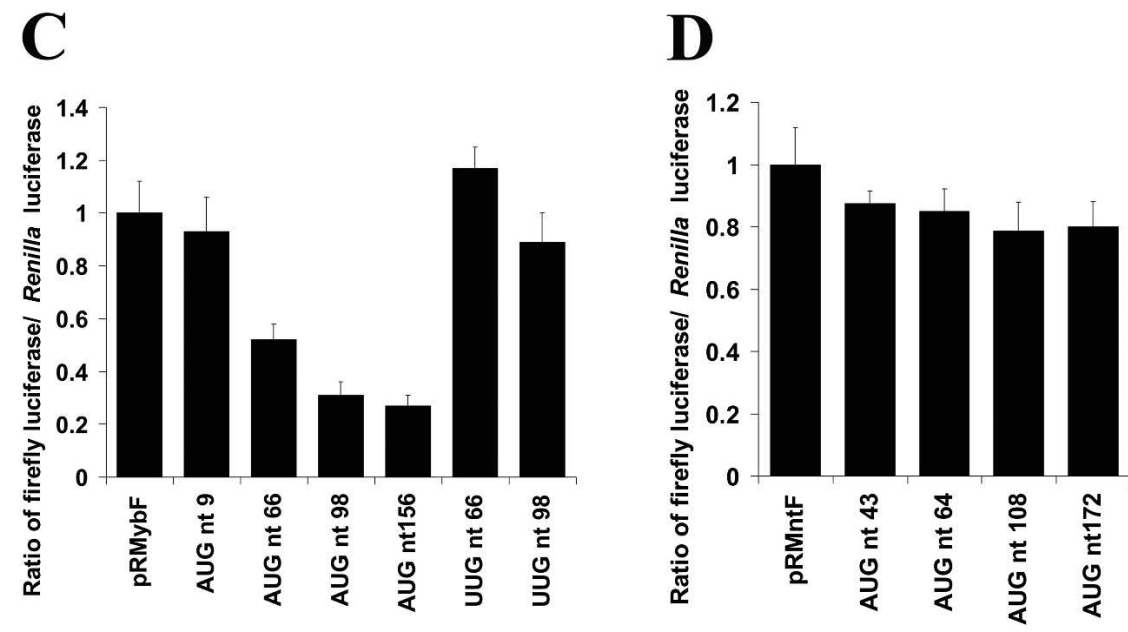

MTG8 5'-UTR

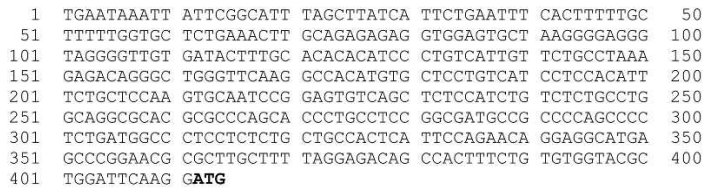

401 TGGAITCAAG GATG

Figure 5. Derivation of structural models for the Myb, Mnt, and MTG8a IRESs. (A) Sequences of the c-myb, Mnt, and MTG8a 5' UTRs. $(B$, panels $i-$ iii) IRES RNA was generated by in vitro transcription and treated with either kethoxal (which modifies unpaired G), DMS (which modifies unpaired A and C), CMCT (which modifies unpaired U), or RNase V1 (which cleaves dsRNA). The modifications observed on PAGE gels were used to constrain the Mfold algorithm and produce a secondary structure prediction. Squares denote the position of modification by DMS, stars denote CMCT, and arrows denote kethoxal. $(B, C, D)$ Locating the ribosome entry window in c-myb, Mnt, and MTG8a IRESs: Site-directed mutagenesis was used to insert out-of-frame AUG codons into the IRESs within the vector pRF. For the c-myb IRES, two AUG codons that affected IRES activity were also mutated to UUG. The position of each mutated sequence is numbered from the A of the ATGG or the U of UUGG. The resulting constructs were transfected into HeLa cells in conjunction with pcDNA3.1/HisB/lacZ. The data are represented as the percentage firefly luciferase activity compared with the activity from the wild-type IRES. As expected, no alteration in the expression of the Renilla cistron was seen in any case (data not shown).

with the result that this region adopts a more open conformation (Fig. 6D, panel i).

\section{Discussion}

It is becoming apparent that certain cellular IRESs are able to function under defined physiological conditions, suggesting that groups of mRNAs that contain these elements are coordinately regulated at the translational level (Johannes et al. 1998; Qin et al. 2004). Coordinated regulation of translation of these mRNAs could be brought about by the presence of a specific binding sequence in the IRESs of these mRNAs for a trans-acting factor. Interaction of this factor with its binding site 
Mitchell et al.

A(i)

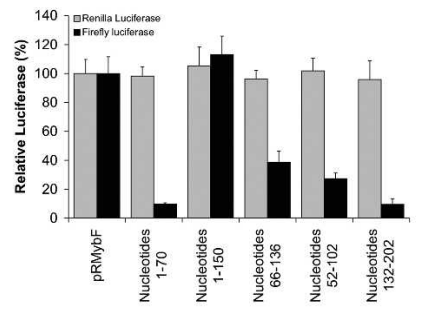

B(i)

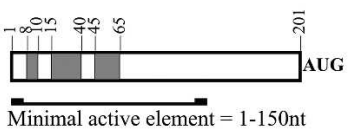

C(i)

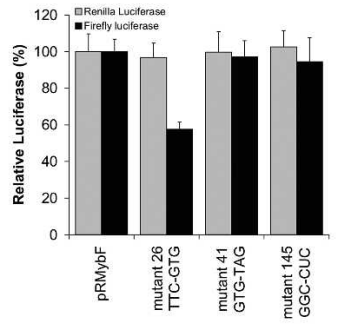

D(i) Myb

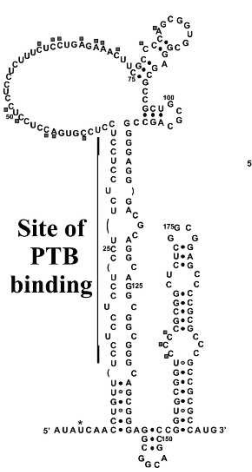

(ii)

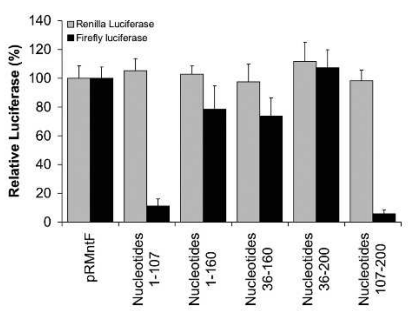

(ii)

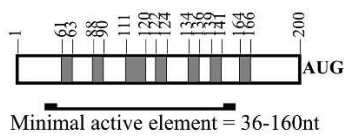

(ii)

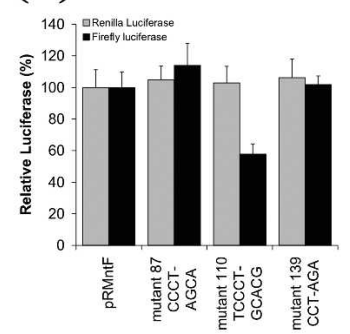

(ii) $\mathrm{Mnt}$ (iii)

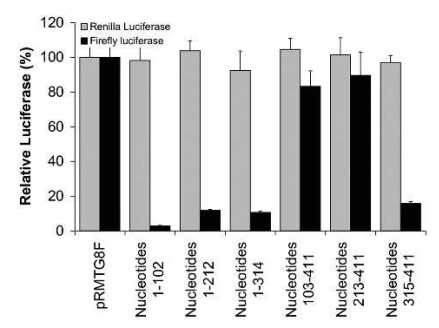

(iii)

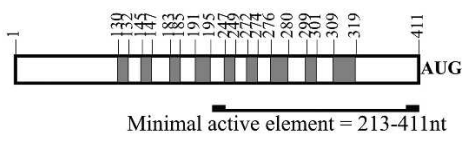

(iii)

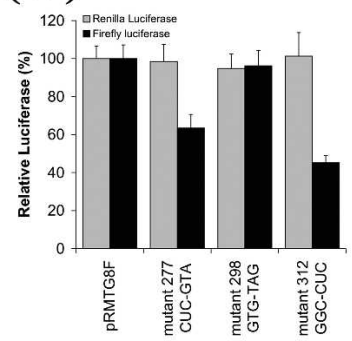

(iii) MTG8a

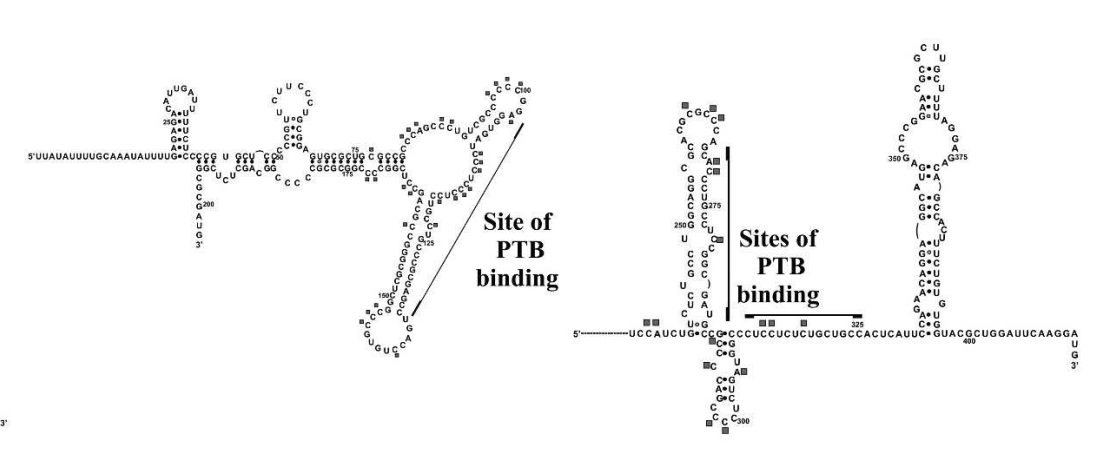

Figure 6. Location of the PTB-binding region on Mnt, c-myb, and MTG8a IRESs. (A, panels i-iii) To define the minimum active element for each IRES, DNA fragments from MTG8a, Myb, and Mnt were amplified using PCR (using oligonucleotides as described in Materials and Methods) and inserted into the dicistronic construct pRF at the EcoRI and NcoI sites. The dicistronic constructs containing the truncated 5' UTR sequences were transfected into HeLa cells. All constructs were cotransfected with pcDNA3.1/ HisB/lacZ. The Renilla luciferase and firefly luciferase activities were determined, normalized to $\beta$-galactosidase activities, and expressed as a percentage of the wild-type IRES activity levels. ( $B$, panels $i$-iii) Positions of the potential PTB-binding regions are marked (in gray) for each IRES, and this overlaps with the minimal active element. All the minimal active fragments bind to PTB in EMSAs (data not shown). (C, panels i-iii) To confirm the PTB binding data the structures were mutated to alter the binding sites as described in the Materials and Methods section. Sites that were mutated are marked in bold, and it can be seen that only those mutations that are located in the PTB-binding regions show any effect on IRES activity. Decreases in firefly luciferase expression of up to $50 \%$ are seen. $(D$, panels $i-i i i)$ The structures of the three IRESs are shown, and the position of the PTB-binding region is marked. PTB binding causes opening of a loop in the c-myb IRES; however, the data suggest that PTB is also binding to the stem that precedes the loop. No changes in the structures of Mnt or MTG8a were observed on PTB binding, and the data suggest that this protein is interacting with both double- and single-stranded regions of RNA. Squares indicate positions of modification by DMS. 
could then stimulate internal ribosome entry on a number of mRNAs simultaneously. There are other examples of this type of regulation; for example, a small stem loop of $\sim 30 \mathrm{nt}$, termed the iron response element (IRE), controls the translation of a subset of mRNAs in response to iron levels, and is central to cellular iron homeostasis (Hentze and Kuhn 1996; Aisen et al. 2001). Control of these mRNAs is mediated by two related cytoplasmic iron regulatory proteins (IRPs) that bind to this element (Hentze and Kuhn 1996; Aisen et al. 2001). Thus one of the central goals of those working in the field of translation is to identify such a sequence motif(s) and proteins that are required for internal ribosome entry on cellular mRNAs. Although secondary structural models for a number of cellular IRESs have now been derived, there are no obvious similarities between these models, even among mRNAs whose protein products have related functions (Le Quesne et al. 2000; Pedersen et al. 2002; Mitchell et al. 2003; Yaman et al. 2003; Jopling et al. 2004; Pickering et al. 2004), although it is entirely possible that the motifs required for coordinated regulation of these elements resides in the tertiary structure.

Recent studies have shown that a small number of eukaryotic IRESs (Gtx and RBM3) contain sequences that are able to recruit ribosomes by binding directly to ribosomal proteins (Chappell et al. 2003, 2004), a mechanism which has also been seen in viral IRESs such as hepatitis C, classic swine fever virus, and bovine viral diarrhoea virus (Pestova et al. 1998; Pestova and Hellen 1999). However, thus far, this method of facilitating translation initiation on IRES elements appears to be unusual among eukaryotic IRESs; in the majority of cases the data suggest that IRES trans-acting factors facilitate ribosome recruitment (Mitchell et al. 2003; Pickering et al. 2004; Thoma et al. 2004). It therefore remains important to determine the proteins that are involved in these interactions.

PTB is a protein that was originally identified as a splicing factor (Garcia-Blanco et al. 1989), and it has been shown that in regulating the alternative splicing of genes, PTB generally acts as a splicing repressor (for review, see Wagner and Garcia-Blanco 2001). In contrast to the data shown in this paper with regard to IRES activation, the optimal binding site for PTB involvement in splicing is single-stranded UCUU within a pyrimidinerich context (Perez et al. 1997a), and exons regulated by PTB are generally associated with one or more such elements. In recent years, PTB has been found to be associated with many viral (Hellen et al. 1994; Ali and Siddiqi 1995; Kaminski et al. 1995, 1998; Niepmann 1996; Schultz et al. 1996; Anwar et al. 2000) and cellular IRESs (Giraud et al. 2001; Nanbru et al. 2001; Pickering et al. 2003, 2004). Our data have previously shown that the Apaf-1 and BAG-1 IRESs have absolute requirements for PTB for function (Mitchell et al. 2003; Pickering et al. 2004). Although the Apaf-1 IRES also requires unr (Mitchell et al. 2003) and the Bag-1 IRES requires PCBP1 (Pickering et al. 2004), these additional factors appear to be necessary to simply remodel the RNA structure to affect PTB binding ability rather than aid in ribosome recruitment. Thus an important question to address is whether PTB is a global IRES trans-acting factor that plays a central role in internal ribosome entry. Therefore, to determine whether the presence of a PTB-binding site alone was sufficient to allow internal ribosome entry, a number of AIRESs were constructed based on the core PTB binding sequence that we identified in the Apaf-1 IRES. This motif consists of a polypyrimidinerich tract that contains repeats of CCU (Fig. 1). The data show that the presence of a PTB-binding site alone is indeed sufficient to mediate internal ribosome entry, providing that the RNA is present as a double-stranded stem (Figs. 1, 2). This was surprising given that PTB was thought to bind to ssRNA, but additional data provide strong evidence that PTB binds preferentially to dsRNA (Fig. 2B). This raises the possibility that PTB or an interacting protein partner could provide a bridge between the IRES-RNA and the ribosome.

To confirm the importance of this motif in internal ribosome entry, and the role of PTB in this process, we analyzed a database of 5' UTRs, the UTRdb (Pesole et al. 2002), for copies of the CCUCC motif. Of the 3000 mRNAs that met these criteria ( 10\% of the database), several were already known to contain IRES elements (cmyc, Stoneley et al. 2000a; AML1, Pozner et al. 2000). Of the mRNAs that we have thus far cloned and examined, all have been found to be PTB-dependent, functional IRESs. The interaction of PTB with three IRESs containing the $(\mathrm{CCU})_{\mathrm{n}}$ motif (c-myb, Mnt, and MTG8a) was studied in more detail. These IRES-RNAs all bind to PTB isoforms and require PTB for function in vitro and in vivo (Figs. 3, 4). Interestingly, the HCV IRES, which has also been shown to interact with PTB in vitro (Anwar et al. 2000) was unaffected by a decrease in PTB levels in vivo, suggesting that this protein is not essential for the activity of this IRES. Structurally the c-myb, Mnt, and MTG8a IRESs are distinct (Fig. 5), but interestingly, in support of our data, which shows that PTB binds to dsRNA, PTB interacted primarily with stem structures in addition to more flexible loops (Fig. 6). This observation is consistent with the PTB binding characteristics demonstrated for the Apaf-1 IRES, where although one CCU motif is present on a single-stranded loop region, which is further modified by unr, the other PTB-binding site is part of a double-stranded stem whose structure is not changed (Mitchell et al. 2003). These data were confirmed by mutational analysis of the Mnt, c-myb, and MTG8a IRESs, which again showed that this polypyrimidine-rich region was essential for the function of these IRESs.

Mechanistically, the way in which PTB may function to recruit the ribosome to these IRES elements is still unclear. Binding assays carried out in this laboratory have led us to conclude that the ribosome probably does not interact directly with PTB (data not shown), and other factors such as HnRNPL (Hahm et al. 1998), Raver (Huttelmaier et al. 2001), or PSF (Peng et al. 2002) may be needed to act as a bridge (Fig. 7).

In summary, given the number of cellular IRESs that 
Mitchell et al.

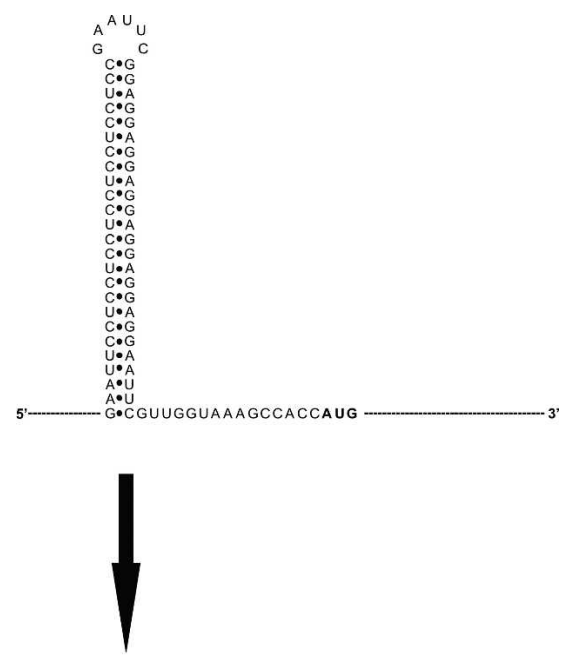

some entry and that this protein is truly a universal IRES-trans-acting factor.

\section{Materials and methods}

Database search

The UTRdb (Pesole et al. 2002) was searched using MEME and MAST (Bailey and Gribskov 1998) or BLAST followed by manual inspection, to identify 5' UTRs of human transcription factor mRNAs that contain at least three copies of the CCUCC motif. The coding sequences for each of the UTRs were retrieved from the EMBL Nucleotide Sequence Database (Kulikov 2004) and used to divide the transcription factors into groups based on the Transfac system of classification (Wingender 2001).

\section{Cell culture and transient transfections}

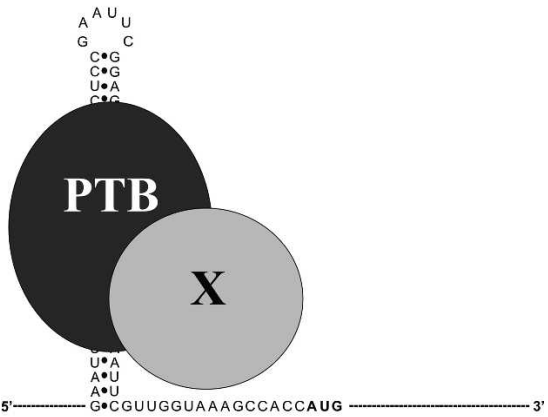

HeLa cells were cultured using conditions described on the ATCC Web page (http://www.atcc.org). Cells were transfected using FuGene 6 (Roche) following the supplier's instructions, or with calcium phosphate (Jordan et al. 1996).

The activities of firefly and Renilla luciferases in in vitro translation reactions or lysates prepared from transfected cells were measured and calculated as previously described (Mitchell et al. 2003) using a Dual-Luciferase reporter assay system (Promega).

\section{SDS-PAGE and Western blotting}

For analysis of PTB, cell extracts (equal cell numbers per lane) were separated by SDS-polyacrylamide gel electrophoresis and electroblotted as described by Mitchell et al. (2001). The antiPTB monoclonal antibody (Chou et al. 2000) was generated by the C.W.J. Smith laboratory (Biochemistry) and used at 1:2000. The anti-actin monoclonal antibody was purchased from Sigma and used at 1:2000. Blots were then incubated with peroxidaseconjugated secondary antibodies raised against mouse or rabbit immunoglobulin and developed using the chemiluminescence reagent "Illumin 8" (generated by Dr. M. Murray, Department of Genetics, University of Leicester, Leicester, UK).

Protein expression

Recombinant PTB-1, PTB-2, PTB-4, nPTB, unr, La, DAP45, and ITAF45 were expressed in BL21(DE3) cells using the pET28a construct. Cells were lysed in phosphate-buffered saline containing $0.1 \%$ Triton X-100, and the His-tagged proteins were purified using a nickel affinity resin.

\section{Reduction of PTB expression by RNAi}

On day 1 , HeLa cells were seeded at a density of $1 \times 10^{5}$ cells per well of a 24-well plate and grown overnight in Dulbecco's modified Eagle medium (DMEM) 10\% fetal calf serum (FCS). On day 2, $6 \mathrm{~mL}$ of a 20-mM stock of siRNAs PTB P1 (AACUUCCAUC AUUCCAGAGAA) and C2 (AAGGUCCGGCUCCCCCAAA UG) (Wagner and Garcia-Blanco 2002) were mixed with $44 \mathrm{~mL}$ Optimem-1 medium (Invitrogen), and then combined with a mixture of $3 \mathrm{~mL}$ Lipofectamine 2000 (Invitrogen) and $12 \mathrm{~mL}$ Optimem- 1 and incubated at room temperature for $25 \mathrm{~min}$. A further $38 \mathrm{~mL}$ of Optimem- 1 was added, and $100 \mathrm{~mL}$ of the mixture transferred to the cells. On day 3, the cells were washed with PBS, 
detached from the plate by addition of $120 \mathrm{~mL}$ of trypsin/EDTA, and incubated at $37^{\circ} \mathrm{C}$ for $2-3 \mathrm{~min}$. DMEM $(380 \mathrm{~mL})$ was added and the cells then split equally between three wells of a 24-well plate, each containing $0.5 \mathrm{~mL}$ of DMEM/FCS. On day 4, the cells were transfected with siRNA as on day 2, except that one-third of the quantities were used per well. On day 4, $0.3 \mathrm{mg}$ of dicistronic reporter per well was transfected using FuGene6 (Roche) according to the manufacturer's instructions. The cells were harvested on day 7 , and luciferase activity determined.

\section{In vitro transcription and in vitro translation}

Vector DNA (pSKUTRL or pUTRF) was linearized by restriction digestion using a site downstream of the sequence of interest. Transcripts were synthesized as described previously (Mitchell et al. 2001). For radiolabeled RNAs, $50 \mu \mathrm{Ci}$ of $[\alpha 32 \mathrm{P}]$ CTP was included in the reactions. After incubation of the reaction for 1 $\mathrm{h}$ at $37^{\circ} \mathrm{C}$, the RNA was isolated and used to prime a rabbit reticulocyte lysate system with a final reaction volume of 12.5 $\mu \mathrm{L}$ as described previously (Mitchell et al. 2001). PTB (0.1 $\mu \mathrm{g}$ ) was added to the translation reactions where indicated. All experiments were performed in triplicate on at least three independent occasions.

\section{EMSAS}

EMSAs were preformed as described previously (Mitchell et al. 2001) using $\sim 23,000 \mathrm{cpm}$ of labeled transcript that was incubated with PTB $(0.2 \mu \mathrm{g})$ at room temperature for $10 \mathrm{~min}$. Loading buffer was added and samples loaded directly onto $5 \%$ or $10 \%$ acrylamide TBE gels that were then electrophoresed at $150 \mathrm{~V}$ for $1 \mathrm{~h}$. Gels were dried and exposed on a PhosphorImager.

\section{PCR mutagenesis}

Introduction of each upstream AUG or UUG and production of the PTB-binding mutants were performed by PCR mutagenesis. PCR was performed using KOD polymerase (Novagen) following the manufacturer's recommendations. The mutant PCR product was digested with SpeI and NcoI, and ligated into pRF.

\section{Northern blot analysis}

Poly A+ mRNA was prepared and analyzed by Northern blotting exactly as described previously (West et al. 1995). DNA probes used for the detection of firefly luciferase mRNA species were also as described (Coldwell et al. 2000).

\section{Secondary structure prediction}

Chemical structure probing was performed exactly as described by Mitchell et al. (2003). Secondary structure predictions were generated using the web implementation of the Mfold algorithm (Zucker 1989) incorporating version 3.0 of the Turner rules (Matthews et al. 1999).

\section{Acknowledgments}

We express our sincere gratitude to Chris Smith and Matthew Wollerton for providing reagents and helpful discussions for the RNAi experiments. This work was supported by grants from the Wellcome Trust (065502/Z/01/Z to S.A.M., and 063233/B/00/Z to M.B.) and the BBSRC (advanced fellowship held by A.E.W., project grant to M.B., project grant funding for K.A.S., M.S.). J.R.E. held an MRC studentship.

\section{References}

Aisen, P., Enns, C., and Wessling-Resnick, M. 2001. Chemistry and biology of eukaryotic iron metabolism. Int. J. Biochem. Cell Biol. 33: 940-959.

Ali, N. and Siddiqui, A. 1995. Interaction of polypyrimidine tract-binding protein with the $5^{\prime}$ noncoding region of the hepatitis $\mathrm{C}$ virus RNA genome and its functional requirement in internal initiation of translation. J. Virol. 69: 63676375.

Anwar, A., Ali, N., Tanveer, R., and Siddiqui, A. 2000. Demonstration of functional requirement of polypyrimidine tractbinding protein by SELEX RNA during hepatitis $\mathrm{C}$ virus internal ribosome entry site-mediated translation initiation. $J$. Biol. Chem. 275: 34231-34235.

Bailey, T.L. and Gribskov, M. 1998. Combining evidence using p-values: Application to sequence homology searches. Bioinformatics 14: 48-54.

Belsham, G.J. and Jackson, R.J. 2000. Translation initiation on Picornavirus RNA. In Translational control of gene expression (eds. N. Sonenberg et al.), pp. 869-900. Cold Spring Harbor Laboratory Press, NY.

Black, D.L., Chan, R., Min, H., Wang, J. and Bell, L. 1998. The electrophoretic mobilty shift assay for RNA binding proteins. In RNA:protein interactions: A practical approach led. C.W.J. Smith), pp. 109-136. Oxford University Press, Oxford.

Bonnal, S., Schaeffer, C., Creancier, L., Clamens, S., Moine, H., Prats, A.C., and Vagner, S. 2003. A single internal ribosome entry site containing a G quartet RNA structure drives fibroblast growth factor 2 gene expression at four alternative translation initiation codons. I. Biol. Chem. 278: 3933039336.

Chappell, S.A. and Mauro, V.P. 2003. The internal ribosome entry site (IRES) contained within the RNA-binding motif protein $3(R b m 3)$ mRNA is composed of functionally distinct elements. J. Biol. Chem. 278: 33793-33800.

Chappell, S.A., Edelman, G.M., and Mauro, V.P. 2004. Biochemical and functional analysis of a 9-nt RNA sequence that affects translation efficiency in eukaryotic cells. Proc. Natl. Acad. Sci. 101: 9590-9594.

Chou, M.Y., Underwood, J.G., Nikolic, J., Luu, M.H.T., and Black, D.L. 2000. Multisite RNA binding and release of polypyrimidine tract binding protein during the regulation of csrc neural-specific splicing. Mol. Cell 5: 949-957.

Coldwell, M.J., Mitchell, S.A., Stoneley, M., MacFarlane, M., and Willis, A.E. 2000 Initiation of Apaf-1 translation by internal ribosome entry. Oncogene 19: 899-905.

Coldwell, M.J., deSchoolmeester, M.L., Fraser, G.A., Pickering, B.M., Packham, G., and Willis, A.E. 2001. The p36 isoform of BAG-1 is translated by internal ribosome entry following heat shock. Oncogene 20: 4095-4100.

Conte, M.R., Grune, T., Ghuman, J., Kelly, G., Ladas, A., Matthews, S., and Curry, S. 2000. Structure of tandem RNA recognition motifs from polypyrimidine tract binding protein reveals novel features of the RRM fold. EMBO $T$. 19: 3132-3141.

Cote, C.A., Gautreau, D., Denegre, J.M., Kress, T.L., Terry, N.A., and Maury, K.L. 1999. A Xenopus protein related to hnRNP I has a role in cytoplasmic RNA localization. Vet. Immunol. Immunopath. 4: 431-437.

García-Blanco, M., Jamison, S., and Sharp, P. 1989. Identification and purification of a 62,000 -dalton protein that binds specifically to the polypyrimidine tract of introns. Genes \& Dev. 3: 1874-1886.

Gil, A., Sharp, P.A., Jamison, S.F., and Garcia-Blanco, M.A. 1991. Characterisation of cDNAs encoding the polypyrimi- 
dine tract binding protein. Genes \& Dev. 5: 1224-1236.

Giraud, S., Greco, A., Brink, M., Diaz, J.J., and Delafontaine, P. 2001. Translation initiation of the insulin-like growth factor I receptor mRNA is mediated by an internal ribosome entry site. J. Biol. Chem. 276: 5668-5675.

Gosert, R., Chang, R., Rojnbrand, R., Yi, M., Sanagr, D., and Lemon, S. 2000. Transient expression of cellular PTB binding protein stimulates cap-independent translation directed by both picornaviral and flaviviral internal ribosome entry sites in vivo. Mol. Cell. Biol. 20: 1583-1585.

Hahm, B., Cho, O.H., Kim, J.E., Kim, Y.K., Kim, J.H., Oh, Y.L., and Jang, S.K. 1998. Polypyrimidine tract-binding protein interacts with HnRNP L. FEBS Lett. 425: 401-406.

Hellen, C.U.T. and Sarnow, P. 2001. Internal ribosome entry sites in eukaryotic mRNA molecules. Genes \& Dev. 15: 1593-1612.

Hellen, C.U.T., Pestova, T.V., Litterst, M., and Wimmer, E. 1994. The cellular polypeptide p57 (pyrimidine tract-binding protein) binds to multiple sites in the poliovirus 5 ' nontranslated region. J. Virol. 68: 941-950.

Hentze, M.W. and Kuhn, L. 1996. Molecular control of vertebrate iron metabolism: mRNA regulatory circuits operated by iron nitric oxide and oxidative stress. Proc. Natl. Acad. Sci. 93: 8175-8182.

Huez, I., Creancier, L., Audiger, S., Gensac, M.-C., Prats, A.-C., and Prats, H. 1998. Two independent internal ribosome entry sites are involved in translation initiation of vascular endothelial growth factor mRNA. Mol. Cell. Biol. 18: 61786190.

Huttelmaier, S., Illenberger, S., Grrosheva, I., Rudieger, M., Singer, R.H., and Jockush, B.M. 2001. Raver 1, a dual compartment protein, is a ligand for $\mathrm{PTB} / \mathrm{hnRNP} 1$ and microfilament attachment proteins. J. Cell Biol. 155: 755-785.

Johannes, G., Carter, M.S., Eisen, M.B., Brown, P.O., and Sarnow, P. 1999. Identification of eukaryotic mRNAs that are translated at reduced cap-binding complex eIF4F concentrations using a cDNA microarray. Proc. Natl. Acad. Sci. 96: 13118-13123.

Jopling, C.L. and Willis, A.E. 2001. N-myc translation is initiated via an internal ribosome entry segment that displays enhanced activity in neuronal cells. Oncogene 20: 26642670.

Jopling, C.L., Spriggs, K.A., Mitchell, S.A., Stoneley, M., and Willis, A.E. 2004. L-Myc protein synthesis is initiated by internal ribosome entry. RNA 10: 287-298.

Jordan, M., Schallhorn, A., and Wurm, F.M. 1996. Transfecting mammalian cells: Optimization of critical parameters affecting calcium-phosphate precipitate formation. Nucleic Acids Res. 24: 596-601.

Kaminski, A. and Jackson, R. 1998. The polypyrimidine tract binding protein requirement for internal initiation of translation of cardiovirus RNAs is conditional rather than absolute. RNA 4: 626-628.

Kaminski, A., Hunt, S.L., Patton, J.G., and Jackson, R.J. 1995. Direct evidence that polypyrimidine tract binding protein (PTB) is essential for internal initiation of translation of encephalomyocarditis virus RNA. RNA 1: 924-938.

Kenan, D.J., Query, C.C., and Keen, J.D. 1991. RNA recognition: Towards identifying determinants for specificity. Trends Biochem. Sci. 16: 214-220.

Kulikov, D. 2004. The EMBL Nucleotide Sequence Database. Nucleic Acids Res. 32: D27-D30.

Le Quesne, J.P.C., Stoneley, M., Fraser, G.A., and Willis, A.E. 2001. Derivation of a structural Model for the c-myc IRES. J. Mol. Biol. 310: 111-126.

Lou, H., Helfman, D.M., Gagel, R.F., and Berget, S.M. 1999.
Polypyrimidine tract-binding protein positively regulates inclusion of an alternative 3 '-terminal exon. Mol. Cell. Biol. 19: $78-85$.

Markovtsov V., Nikolic J., Goldman J.A., Tyrck C.T., Chou M-Y., and Black, D.L. 2000. Cooperative assembly of an hnRNP complex induced by a tissue-specific homologue of PTB. Mol. Cell. Biol. 20: 7463-7479.

Matthews, D., Sabina, J., Zuker, M., and Turner, D. 1999. Expanded sequence dependence of thermodynamic parameters improves prediction of RNA secondary structure. J. Mol. Biol. 288: 911-940.

Mitchell, S.A., Brown, E.C., Coldwell, M.J., Jackson, R.J., and Willis, A.E. 2001. Protein factor requirements of the Apaf-1 internal ribosome entry segment: Roles of polypyrimidine tract binding protein and upstream of N-ras. Mol. Cell. Biol. 21: 3364-3374.

Mitchell, S.A., Spriggs, K.A., Coldwell, M.J., Jackson, R.J., and Willis, A.E. 2003. The Apaf-1 internal ribosome entry segment attains the correct structural conformation for function via interactions with PTB and unr. Mol. Cell 11: 757711.

Nanbru,C., Prats, A.-C., Droogmans, L., Defrance, P., Huez, G., and Kruys, V. 2001. Translation of the human c-myc P0 tricistronic mRNA involved two independent internal ribosome entry sites. Oncogene 20: 4270-4280.

Niepmann, M. 1996. Porcine polypyrimidine tract-binding protein stimulates translation initiation at the internal ribosome entry site of foot-and-mouth disease virus. FEBS Lett. 388: $39-42$.

Pain, V.M. 1996. Initiation of protein synthesis in eukaryotic cells. Eur. J. Biochem. 236: 747-771.

Pedersen, S.K., Christiansen, J., Hansen, T.V., Larsen, M.R., and Nielsen, F.C. 2002. Human insulin-like growth factor II leader 2 mediates internal initiation of translation. Biochem. I. 363: 37-44.

Peng, R., Dye, B.T., Perez, I., Barnard, D.C., Thompson, A.B., and Patton, J.G. 2002. PSF and p54(nrb) bind a conserved stem in U5 snRNA. RNA 8: 1334-1347.

Perez, I., Lin, C.H., McAfee, J.G., and Patton, J.G. 1997a. Mutation of PTB binding sites causes misregulation of alternative 3' splice site selection in vivo. RNA 3: 764-778.

Perez, I., McAfee, J.G., and Patton, J.G. 1997b. Multiple RRMs contribute to RNA binding specificity and affinity for polypyrimidine tract binding protein. Biochemistry 36: 1188111890.

Pesole G., Liuni S., Grillo G., Licciulli F., Mignone F., Gissi C., and Saccone, C. 2002. UTRdb and UTRsite: Specialized database of sequences and functional elements of $5^{\prime}$ and $3^{\prime}$ untranslated regions of eukaryotic mRNAs. Update 2002. Nucleic Acids Res. 30: 335-340.

Pestova, T.V. and Hellen, C.U.T. 1999. Internal initiation of bovine viral diarrhea virus. Virology 258: 249-256.

Pestova, T.V., Shatsky, I.N., Fletcher, S.P., Jackson, R.J., and Hellen, C.U.T. 1998 A prokaryotic-like mode of cytoplasmic eukaryotic ribosome binding to the initiation codon during internal translation initiation of hepatitis $\mathrm{C}$ and classical swine fever virus. Genes \& Dev. 12: 67-83.

Pickering, B.M., Mitchell, S.A., Evans, J.R., and Willis, A.E. 2003. Polypyrimidine tract binding protein and poly $(\mathrm{rC})$ binding protein 1 interact with the BAG-1 IRES and stimulate its activity in vitro and in vivo. Nucleic Acids Res. 31: 639-646.

Pickering, B.M., Mitchell, S.A., Spriggs, K.A., Stoneley, M., and Willis, A.E. 2004. BAG-1 internal ribosome entry segment activity is promoted by structural changes mediated by poly (rC) binding protein 1 and recruitment of polypyrimidine 
tract binding protein 1. Mol. Cell. Biol. 24: 5595-5604.

Polydorides, A.D., Okano, H.J., Yang, Y.Y.L., Stefani, G., and Darnell, R.B. 2000. A brain-enriched polypyrimidine tractbinding protein antagonizes the ability of Nova to regulate neuron-specific alternative splicing. Proc. Natl. Acad. Sci. 97: 6350-6355.

Pozner, A., Goldenberg, D., Negreanu, V., Le, S.Y., Elroy-Stein, O., Levanon, D., and Groner, Y. 2000. Transcription-coupled translation control of AML1/RUNX1 is mediated by capand internal ribosome entry site-dependent mechanisms. Mol. Cell. Biol. 20: 2297-2307.

Qin, X.L. and Sarnow, P. 2004. Preferential translation of internal ribosome entry site-containing mRNAs during the mitotic cycle in mammalian cells. J. Biol. Chem. 279: 1372113728.

Schultz, D.E., Hardin, C.C., and Lemon, S.M. 1996. Specific interaction of glyceraldehyde 3-phosphate dehydrogenase with the $5^{\prime}$-nontranslated RNA of Hepatitis A virus. J. Biol. Chem. 271: 14134-14142.

Simpson, P.J., Monie, T.P., Szendroi, A., Davydova, N., Tyzack, J.K., Conte, M.R., Read, C.M., Cary, P.D., Svergun, D.I., Konarev, P.V., et al. 2004. Structure and RNA interactions of the N-terminal RRM domains of PTB. Structure 12: 16311643.

Singh, R., Valcarcel, J., and Green, M.R. 1995. Distinct binding specificities and functions of higher eukaryotic polypyrimidine tract-binding proteins. Science 268: 1173-1176.

Stein, I., Itin, A., Einat, P., Skaliter, R., Grossman, Z., and Keshet, E. 1998. Translation of vascular endothelial growth factor mRNA by internal ribosome entry: Implications for translation under hypoxia. Mol. Cell. Biol. 18: 3112-3119.

Stoneley, M., Chappell, S., Jopling, C., Dickens, M., MacFarlane, M., and Willis, A. 2000a. c-Myc protein synthesis is initiated from the internal ribosome entry segment during apoptosis. Mol. Cell. Biol. 20: 1162-1169.

Stoneley, M., Subkhankulova, T., Le Quesne, J.P.C., Coldwell, M.J., Jopling, C.L., Belsham, G.J., and Willis, A.E. 2000 b. Analysis of the c-myc IRES; a potential role for cell-type specific trans-acting factors and the nuclear compartment. Nucleic Acids Res. 28: 687-694.

Stoneley, M., Spencer, J.P., and Wright, S.C. 2001. An internal ribosome entry segment in the $5^{\prime}$ untranslated region of the mnt gene. Oncogene 20: 893-897

Subkhankulova, T., Mitchell S.A., and Willis, A.E. 2001. Internal ribosome entry segment mediated initiation of $\mathrm{c}-\mathrm{Myc}$ protein synthesis following genotoxic stress. Biochem. I. 359: 183-192.

Thoma, C., Bergamini, G., Galy, B., Hundsdoerfer, P., and Hentze, M.W. 2004. Enhancement of IRES-mediated translation of the c-myc and BiP mRNAs by the poly(A) tail is independent of intact eIF4G and PABP. Mol. Cell 15: 925935.

Tillmar, L. and Welsh, N.J. 2004. Glucose-induced binding of the polypyrimidine tract-binding protein (PTB) to the $3^{\prime}$-untranslated region of the insulin mRNA (ins-PRS) is inhibited by rapamycin. Mol. Cell Biochem. 260: 85-90.

Wagner, E.J. and Garcia-Blanco, M.A. 2001. Polypyrimidine tract binding protein antagonizes exon definition. Mol. Cell. Biol. 21: 3281-3288.

- 2002. RNAi-mediated PTB depletion leads to enhanced exon definition. Mol. Cell 10: 943-949.

West, M.J., Sullivan, N.F., and Willis, A.E. 1995. Translational upregulation of the c-myc oncogene in Bloom's syndrome cell lines. Oncogene 11: 2515-2524.

Wingender, E. 2001. The TRANSFAC system on gene expression regulation. Nucleic Acids Res. 29: 281-283.
Wollerton, M.C., Gooding, C., Wagner, E.J., Garcia-Blanco, M.A., and Smith, C.W.J. 2004. Autoregulation of polypyrimidine tract binding protein by alternative splicing leading to nonsense-mediated decay. Mol. Cell 13: 91-100.

Yaman, I., Fernandez, J., Liu, H.Y., Caprara, M., Komar, A.A., Koromilas, A.E., Zhou, L.Y., Snider, M.D., Scheuner, D., Kaufman, R.J., et al. 2003. The zipper model of translational control: A small upstream ORF is the switch that controls structural remodeling of an mRNA leader. Cell 113: 519531.

Zucker, M. 1989. On finding all suboptimal foldings of an RNA molecule. Science 244: 48-52. 


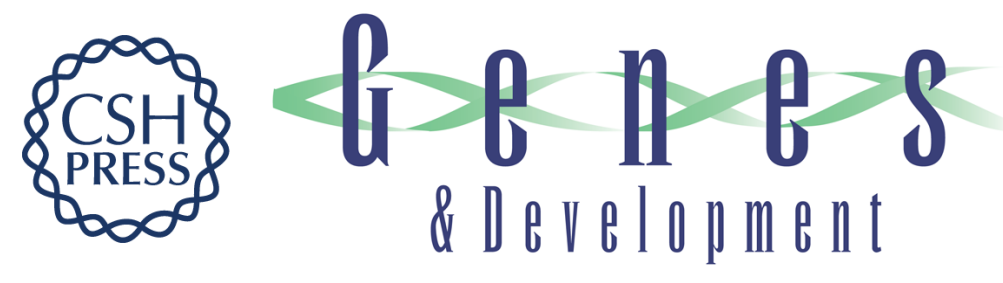

\section{Identification of a motif that mediates polypyrimidine tract-binding protein-dependent internal ribosome entry}

Sally A. Mitchell, Keith A. Spriggs, Martin Bushell, et al.

Genes Dev. 2005, 19:

Access the most recent version at doi:10.1101/gad.339105

Supplemental http://genesdev.cshlp.org/content/suppl/2005/07/12/19.13.1556.DC1
Material

References This article cites 68 articles, 35 of which can be accessed free at:

http://genesdev.cshlp.org/content/19/13/1556.full.html\#ref-list-1

License

Email Alerting

Receive free email alerts when new articles cite this article - sign up in the box at the top

Service

right corner of the article or click here.

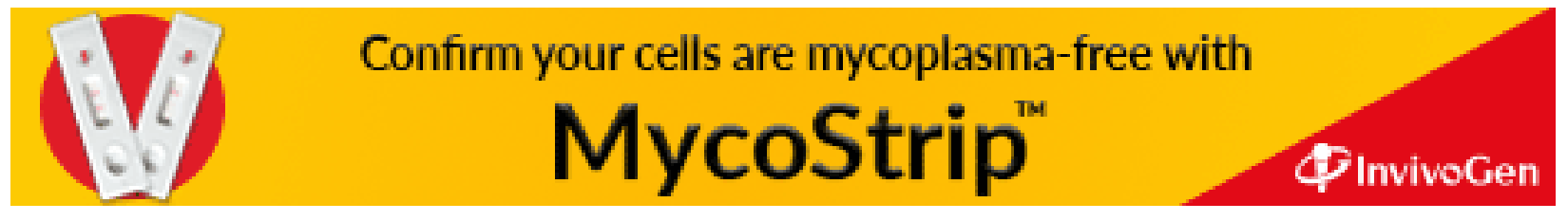

